\title{
Grupos de Witt sobre Domínios de Dedekind
}

\author{
NELSON Fernando Inforzato
}

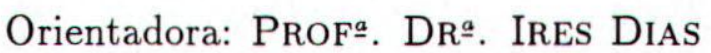

Dissertação apresentada ao Instituto de Ciências Matemáticas e de Computação-USP, como parte dos requisitos para obtenção do título de "Mestre em Ciências - Área: Matemática".

USP - São Carlos

1998 
"À minha família." 
Inicialmente agradeço À Deus, por ter me dado forças suficientes a fim de superar os obstáculos e conquistar o meu objetivo. Agradeço à minha orientadora Prof ${ }^{a}$ Ires pela sua grande dedicação, paciência e amizade; aos meus pais e minha irmã que me apoiaram e incentivaram todo o tempo; à minha noiva Adriana, que esteve sempre ao meu lado com seu amor, carinho e amizade; aos amigos que aqui conquistei ; à Alexandra, pela sua grande amizade; a todos meus professores pelos conhecimentos transmitidos, em especial ao Prof. Nelo da Silva Allan; ao Prof. Hermínio Cassago Júnior pelo auxílio no trabalho de digitação; à CAPES pelo financiamento parcial deste trabalho; ao Departamento de Matemática da Universidade Estadual de Londrina pela liberação parcial de minhas atividades docentes; e a todos que direta ou indiretamente contribuiram para o andamento e conclusão deste trabalho. 


\section{Resumo}

Neste trabalho apresentamos relações entre os grupos de Witt dos espaços bilineares sobre um domínio de Dedekind, dos espaços bilineares sobre seu corpo de frações e dos espaços bilineares definidos em módulos de torção sobre um domínio de Dedekind. Como aplicação destas relações, determinamos o grupo de Witt dos espaços bilineares sobre o anel dos números inteiros. 


\begin{abstract}
In this work we present some relations among the Witt groups of bilinear spaces over Dedekind domain, of bilinear spaces over your quotient field and of bilinear spaces on torsion modules over a Dedekind domain. As one aplication of this relations, we stablish the Witt group of bilinear spaces over the integer numbers.
\end{abstract}




\section{Índice}

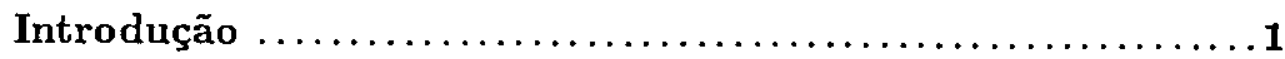

1 Domínios de Dedekind $\ldots \ldots \ldots \ldots \ldots \ldots \ldots \ldots \ldots \ldots \ldots$

2 Ideais Fracionários e Domínios de Dedekind $\ldots \ldots \ldots \ldots \ldots 10$

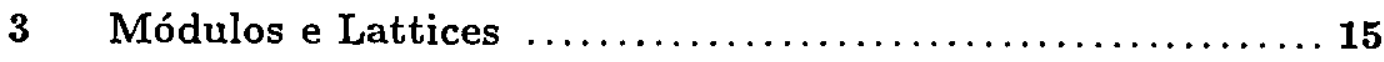

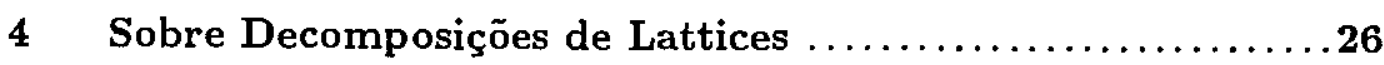

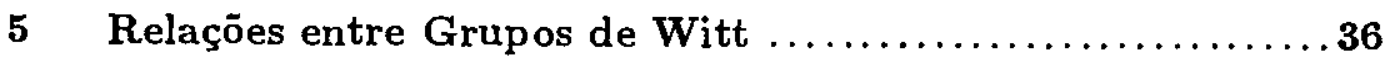

6 Grupos de Witt de Anéis de Valorização Discreta ....... 47

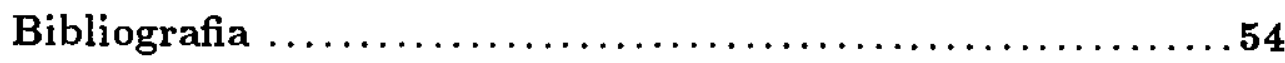




\section{Introdução}

Por um longo tempo - pelo menos de Fermat até Minkowski - a teoria de formas quadráticas era uma parte da teoria de números. Grande parte dos melhores trabalhos em teoria de números dos séculos XVIII e XIX foram relacionados com problemas sobre formas quadráticas. Neste sentido, Minkowski, Siegel, Hasse Eichler e muitos outros criaram a teoria "aritmética" de formas quadráticas, a qual foi objeto de vários artigos e alguns livros clássicos que, dentre os quais destacamos o de O. T. O'Meara [OM].

Em 1937, Witt abriu um novo capítulo na teoria de forma quadráticas, sua mais frutífera idéia foi considerar não simplesmente formas quadráticas "individuais", mas o conjunto de todas as formas quadráticas sobre um dado corpo base e, a partir disto construir um objeto algébrico, chamado o anel de Witt, que se tornou o principal objeto da teoria toda.

Neste trabalho nos propomos a estudar os fatos básicos da teoria aritmética das formas quadráticas sobre um domínio de Dedekind, bem como as relações entre os grupos de Witt dos espaços bilineares sobre um domínio de Dedekind, 
dos espaços bilineares sobre seu corpo de frações e dos espaços bilineares de torção.

Para tanto, nas seções 1 e 2, apresentamos a definição e as propriedades básicas de domínios de Dedekind, culminando com a caracterização de tais domínios via ideais fracionários.

Nas seções 3 e 4, apresentamos a noção de lattices e/ou módulos sobre domínios de Dedekind, bem como a noção de base para lattices e teoremas de decomposição de lattices.

Nas duas seções restantes, tratamos do estudo de formas bilineares simétricas não singulares sobre domínios de Dedekind, dos grupos de Witt destas formas e, algumas relaçōes entre eles. Finalizamos este trabalho, caracterizando os grupos de Witt de anéis de valorização discreta e, usando os resultados apresentados, determinamos o grupo de Witt dos espaços bilineares sobre o anel dos números inteiros. 


\section{Domínios de Dedekind}

Em todo este trabalho, os anéis considerados serão comutativos com elemento identidade 1.

Nesta seção apresentaremos a definição, alguns exemplos e propriedades básicas de domínios de Dedekind. Para tanto, usaremos alguns resultados básicos de álgebra comutativa, dos quais, alguns serão somente citados e, cujas demonstrações podem ser encontradas, por exemplo, em [Ati].

Proposição 1.1 Seja A um domínio Noetheriano de dimensão 1 (dimensão de Krull). Então todo ideal não nulo $\mathcal{I}$ de A pode ser expresso, de maneira única, como um produto de ideais primários cujos radicais são todos distintos.

Dem.: Ver (9.1) em [Ati].

Seja $A$ um domínio Noetheriano de dimensão 1 em que cada ideal primário é potência de um ideal primo. Pela proposição anterior, em $A$ vale a fatoração única de ideais não nulos como produto de ideais primos. Localizando $A$ com respeito a um ideal primo $\mathcal{P}$ não nulo, obteremos um domínio Noetheriano local de dimensão $1, A_{\mathcal{P}}=S^{-1} A$, onde $S=A-\mathcal{P}$. Consequentemente, aplicando 
a proposição (1.1) temos que em $A_{\mathcal{P}}$, todo ideal não nulo é potência do ideal maximal $\mathcal{P} A_{\mathcal{P}}$.

Definição 1.2 Seja K um corpo. Uma valorização discreta em K é uma aplicação $v$ de $K^{*}$ sobre $\mathbb{Z}$ (onde $K^{*}=K-\{0\}$ é o grupo multiplicativo de $K$ ) tal que, para todo $\alpha, \beta \in K^{*}$ temos:

i) $v(\alpha \beta)=v(\alpha)+v(\beta)$; isto é, $v$ é um homomorfismo de grupos abelianos.

ii) $v(\alpha+\beta) \geq \min \{v(\alpha), v(\beta)\}$.

Algumas vezes é conveniente extendermos $v$ à todo $K$ pondo $v(0)=+\infty$. É fácil ver que o conjunto $A_{v}=\{\alpha \in K ; v(\alpha) \geq 0\}$ é um subanel de $K$, chamado $o$ anel de valorização de $v$. Vejamos alguns exemplos de anéis de valorização.

Exemplo 1.3 Seja $K=\mathbb{Q}$ o corpo dos números racionais. Dado um número primo $p$, temos que cada $\alpha \in \mathbb{Q}, \alpha \neq 0$, pode ser escrito, de maneira única, na forma $p^{k} \beta$, onde $k \in \mathbb{Z}$ e, ambos, numerador e denominador de $\beta$ são primos com $p$. Definindo $v_{p}(\alpha)=k$, é fácil ver que $v_{p}$ é uma valorização discreta em $\mathbb{Q}$ e que o anel de valorização de $v_{p}$ é o anel $\mathbb{Z}_{(p)}=\left\{\frac{a}{b} \in \mathbb{Q} ; p / b\right\}$.

Exemplo 1.4 Seja $K=F[x]$, o anel de polinômios a uma variável sobre um corpo $F$. Dado um polinômio irredutível $f \in F[x]$, podemos definir $v_{f}$ de maneira análoga ao exemplo anterior, e neste caso, o anel de valorização de $v_{f}$ é a localização de $F[x]$ com respeito ao ideal primo $(f)$, ou seja, $A_{v_{f}}=F[x]_{(f)}$.

Dizemos que um domínio integral $A$ é um anel de valorização discreta se, existe uma valorização discreta $v$, em seu corpo de frações $K$, tal que $A$ é o anel de valorização de $v$. Se $A$ é um anel de valorização discreta, é fácil ver que $A$ é um anel local, com ideal maximal $\mathfrak{m}=\{\alpha \in K ; v(\alpha)>0\}$, ou seja, $A^{*}$ o grupo 
das unidades de $A$, é o conjunto $\{\alpha \in A ; v(\alpha)=0\}$. Se dois elementos $\alpha, \beta$ de $A$ tem o mesmo valor, isto é, $v(\alpha)=v(\beta)$, então $v\left(\alpha \beta^{-1}\right)=0$, ou seja, $u=\alpha \beta^{-1}$ é uma unidade em $A$. Assim os ideais principais $(\alpha)$ e $(\beta)$ de $A$, são iguais.

Seja $\mathcal{I}$ um ideal de $A$. Então existe um menor inteiro positivo $t$ tal que $v(\alpha)=t$, para algum $\alpha \in \mathcal{I}$. Mais ainda, $\mathcal{I}$ contém todo $\beta \in A \operatorname{com} v(\beta) \geq t$ pois, se $v(\beta) \geq t$, então $\beta=p^{t} \frac{a^{\prime}}{b^{\prime}}$ e, como $\alpha=p^{t} \frac{a}{b}$, podemos escrever $\beta=\alpha \beta^{\prime}$, para algum $\beta^{\prime} \in A$, o que mostra que $\beta \in \mathcal{I}$. Portanto, os únicos ideais não nulos de $A$ são os ideais $\mathfrak{m}_{t}=\{\beta \in A ; v(\beta) \geq t\}$. Estes ideais formam uma única cadeia estritamente decrescente $\mathfrak{m}=\mathfrak{m}_{1} \supset \mathfrak{m}_{2} \supset \mathfrak{m}_{3} \supset \cdots$ e, consequentemente, $A$ é um anel Noetheriano local. Adicionalmente, desde que $v: K^{*} \longrightarrow \mathbb{Z}$ é sobrejetora, existe $\alpha \in \mathfrak{m}$ tal que $v(\alpha)=1$. É fácil ver que $\mathfrak{m}=(\alpha)$. Então $\mathfrak{m}_{k}=\left(\alpha^{k}\right)$, para todo $k \geq 1$. Logo m é o único ideal primo não nulo de $A$. Mostramos com isso que todo anel de valorização discreta é um domínio Noetheriano local de dimensão 1 em que cada ideal não nulo é uma potência do ideal maximal.

Consideremos agora um domínio Noetheriano local $A$, de dimensão 1 , com ideal maximal $\mathfrak{m}$. Desde que $A$ é um domínio de dimensão 1 , temos que todo ideal primo não nulo de $A$ é maximal. Assim, o único ideal primo não nulo de $A$ é $\mathfrak{m}$. Portanto, se $\mathcal{I}$ é um ideal não trivial de $A$, então o radical de $\mathcal{I}$, $r(\mathcal{I})=\left\{\alpha \in A ; \alpha^{n} \in \mathcal{I}\right.$ para algum $\left.n>0\right\}=\mathfrak{m}$. Logo $A$ satisfaz as equivalências de (7.16) em [Ati]. Se para algum $n \geq 0, \mathfrak{m}^{n}=\mathfrak{m}^{n+1}$, então pelo lema de Nakayama, temos que $\mathfrak{m}^{n}=0$, ou seja, $\mathfrak{m}^{n} \subseteq \mathcal{P}$ para todo ideal primo $\mathcal{P}$ de $A$. Tomando os radicais, obtemos $\mathfrak{m}=r\left(\mathfrak{m}^{n}\right) \subseteq r(\mathcal{P})=\mathcal{P}$. Mas $A$ é um domínio de dimensão 1, então $A$ tem pelo menos um ideal primo não nulo. Assim temos:

(A) Se $\mathcal{I}$ é um ideal não trivial de $A$, então $\mathcal{I}$ é m-primário e $\mathcal{I} \supseteq \mathfrak{m}^{n}$ para algum $n \geq 0$. 
(B) $\mathfrak{m}^{n} \neq \mathfrak{m}^{n+1}$, para todo $n \geq 0$.

O próximo teorema caracteriza os domínios Noetherianos discutidos acima.

Teorema 1.5 Sejam A um domínio Noetheriano local de dimensão $1, \mathfrak{m}$ seu ideal maximal e $\bar{K}=A / \mathfrak{m}$ seu corpo residual. Então são equivalentes:

i) A é um anel de valorização discreta;

ii) A é integralmente fechado;

iii) $\mathfrak{m}$ é um ideal principal;

iv) $\operatorname{dim}_{\bar{K}}\left(\mathfrak{m} / \mathfrak{m}^{2}\right)=1$

v) Todo ideal não nulo é potência de m;

vi) Existe $\alpha \in A$ tal que todo ideal näo nulo é da forma $\left(\alpha^{t}\right)$, com $t \geq 0$.

Dem.: i) $\Rightarrow$ ii). Se $A$ é um anel de valorização discreta, então existe uma valorização discreta $v$ no corpo de frações $K$ de $A$ tal que $A=\{\alpha \in K ; v(\alpha) \geq 0\}$. Assim dado $\alpha \in K, \alpha \neq 0$, temos que $\alpha \in A$ ou $\alpha^{-1} \in A$. Se $\alpha$ é inteiro sobre $A$, então existem $a_{1}, \ldots, a_{n} \in A$ tais que

$$
\alpha^{n}+a_{1} \alpha^{n-1}+\cdots+a_{n}=0 .
$$

Se $\alpha \notin A$, então $\alpha^{-1} \in A$ e, $\alpha=-\left(a_{1}+a_{2} \alpha^{-1}+\cdots+a_{n} \alpha^{1-n}\right) \in A$, o que mostra ii).

ii) $\Rightarrow$ iii). Seja $a \in \mathfrak{m}$ e $a \neq 0$. De (A), existe um inteiro $n \geq 1$, tal que $\mathfrak{m}^{n} \subseteq(a)$ e $\mathfrak{m}^{n-1} \nsubseteq(a)$. Escolhendo $b \in \mathfrak{m}^{n-1}$ tal que $b \notin(a)$ e tomando $\alpha=\frac{a}{b} \in K$, temos que $\alpha^{-1} \notin A$ (pois $b \notin(a)$ ). Assim $\alpha^{-1}$ não é inteiro sobre $A$. Consequentemente, $\alpha^{-1} \mathfrak{m} \nsubseteq \mathfrak{m}$ pois caso contrário, $\mathfrak{m}$ seria um $A\left[\alpha^{-1}\right]$-módulo fiel, finitamente gerado 
como um $A$-módulo, o que contradiz as equivalências de (5.1) em [Ati]. Mas $\alpha^{-1} \mathfrak{m} \subseteq A$ pela contrução de $\alpha$. Assim $\alpha^{-1} \mathfrak{m}=A$, ou seja, $\mathfrak{m}=A \alpha=(\alpha)$.

iii) $\Rightarrow$ iv). Desde que $\mathfrak{m}$ é principal, temos que $\operatorname{dim}_{R}\left(\mathfrak{m} / \mathfrak{m}^{2}\right) \leq 1$ e, de $(\mathbf{B})$ $\mathfrak{m} / \mathfrak{m}^{2} \neq 0$, o que implica $\left.i v\right)$.

iv) $\Rightarrow v$ ). Seja $\mathcal{I}$ um ideal não trivial de $A$. De (A), temos que $\mathcal{I} \supseteq \mathfrak{m}^{n}$, para algum $n \geq 0$, então $\mathcal{I}$ é um ideal de $A / \mathfrak{m}^{n}$ que é um anel noetheriano de dimensão zero. Agora de (8.8) em [Ati], obtemos que $\mathcal{I}$ é uma potência de $\mathfrak{m}$.

v) $\Rightarrow$ vi). De (B), $\mathfrak{m} \neq \mathfrak{m}^{2}$. Assim, existe $\alpha \in \mathfrak{m}$ tal que $\alpha \notin \mathfrak{m}^{2}$. Mas por hipótese, $(\alpha)=\mathfrak{m}^{r}$, para algum $r \geq 0$. Logo $r=1$ e $(\alpha)=\mathfrak{m}$, o que implica v).

vi) $\Rightarrow$ i). Claramente $(\alpha)=\mathfrak{m}$ e, novamente de $(\mathbf{B}),\left(\alpha^{t}\right) \neq\left(\alpha^{t+1}\right)$, para todo $t \geq 0$. Assim, para $a \in A, a \neq 0$, temos $(a)=\left(\alpha^{t}\right)$ para exatamente um valor de $t$. Definindo $v(a)=t$ e, extendendo $v$ à $K^{*}$ por $v\left(a b^{-1}\right)=v(a)-v(b)$, temos que $v$ é uma valorização discreta em $K$ e $A$ é o seu anel de valorização.

Para domínios Noetherianos de dimensão 1, temos as seguintes equivalências: Teorema 1.6 Seja A um dominio Noetheriano de dimensão 1. Então, são equivalentes:

i) A é integralmente fechado;

ii) Todo ideal primário de A é uma potência de primo;

iii) Para todo ideal primo $\mathcal{P} \neq 0$ de $A$, o anel local $A_{\mathcal{P}}$ é um anel de valorização discreta.

Dem.: $i) \Leftrightarrow$ iii). Segue do teorema (1.5) e do fato que ser integralmente fechado é uma propriedade local. 
ii) $\Leftrightarrow$ iii). Segue do teorema (1.5) e do fato que ideais primários e potências de ideais comportam-se bem sob localizações (ver (4.8) e (3.11) em [Ati]).

Um anel satisfazendo as condições do teorema acima é dito ser um Dominio de Dedekind.

Como consequência imediata dos dois teoremas anteriores temos:

Corolário 1.7 Num dominio de Dedekind todo ideal não nulo tem uma fatoração única como um produto de ideais primos.

Vejamos agora alguns exemplos de domínios de Dedekind.

Exemplo $1.8 O$ anel dos inteiros $\mathbb{Z}$ é um domínio de Dedekind. Mais geralmente, todo domínio de ideais principais $A$ é um domínio de Dedekind. De fato, desde que todo ideal é principal e, consequentemente finitamente gerado, temos que $A$ é Noetheriano. $O$ anel $A$ tem dimensão 1 , pois num domínio de ideais principais, todo ideal primo não nulo é maximal.

Mais ainda, para todo ideal primo $\mathcal{P} \neq 0$ de $A$, o anel local $A_{\mathcal{P}}$ é também um domínio de ideais principais. Logo seu único ideal maximal é principal. Desde que $A_{\mathcal{P}}$ tem dimensão 1 , temos do teorema (1.5) que $A_{\mathcal{P}}$ é um anel de valorização discreta para todo ideal primo não nulo $\mathcal{P}$ de $A$. Assim, $A$ é um domínio de Dedekind.

Exemplo 1.9 Seja $K$ um corpo de números algćbricos, isto é, uma extensão algébrica finita de $\mathbb{Q}$. Seu anel de inteiros $A$, é o fecho integral de $\mathbb{Z}$ em $K$. Por exemplo, se $K=\mathbb{Q}(i)$, então $A=\mathbb{Z}[i]$, o anel dos inteiros de Gauss. Então, como veremos no próximo teorema, $A$ é um domínio de Dedekind. 
Teorema $1.10 O$ anel de inteiros $A$ de um corpo de números algébricos $K$ é um dominio de Dedekind.

Dem.: Desde que $\mathbb{Q}$ tem característica zero, temos que $K$ é uma extensão separável de $\mathbb{Q}$. Logo existe uma base $\left\{v_{1}, \ldots, v_{n}\right\}$ de $K$ sobre $\mathbb{Q}$ tal que $A \subseteq$ $\sum \mathbb{Z} v_{j}$ (ver (5.17) em [Ati]). Assim, $A$ é finitamente gerado como $\mathbb{Z}$-módulo e portanto Noetheriano. Mais ainda, como $A$ é o anel de inteiros de $K$, temos que $A$ é igual ao seu fecho integral em $K$ ○ que implica que $A$ é integralmente fechado. Logo, para mostrarmos que $A$ é um domínio de Dedekind, é suficiente mostrarmos que $A$ tem dimensão 1 ou equivalentemente, que todo ideal primo não nulo de $A$ é maximal. Seja $\mathcal{P}$ um ideal primo não nulo de $A$. Desde que $A$ é uma extensão inteira de $\mathbb{Z}$, se $\mathcal{P} \cap \mathbb{Z}=(0)=(0) \cap \mathbb{Z}$, temos de (5.9) em [Ati] que $\mathcal{P}=(0)$. Logo $\mathcal{P} \cap \mathbb{Z} \neq(0)$ e é um ideal primo de $\mathbb{Z}$, portanto maximal. Então, novamente de (5.8) em [Ati], temos que $\mathcal{P}$ é um ideal maximal de $A$, como queríamos. 


\section{Ideais Fracionários e Domínios de Dedekind}

Nesta seção apresentaremos uma caracterização de domínios de Dedekind por ideais fracionários.

Sejam $A$ um domínio e $K$ seu corpo de frações. Um $A$-submódulo $\mathcal{F}$ de $K$ é dito ser um ideal fracionário de $A$ se $\alpha \mathcal{F} \subseteq A$, para algum $\alpha \neq 0$ em $A$. Em particular os ideais "ordinários" de $A$, são ideais fracionários, pois basta tomar $\alpha=1$. Qualquer elemento $u \in K$ gera um ideal fracionário, denotado por $(u)$, ou $A u$, e chamado principal. Se $\mathcal{F}$ é um ideal fracionário, o conjunto de todo $\alpha \in K$ tal que $\alpha \mathcal{F} \subseteq A$ é denotado por $(A: \mathcal{F})$.

Todo $A$-submódulo finitamente gerado $\mathcal{F}$ de $K$ é um ideal fracionário. De fato, se $\mathcal{F}$ é gerado por $\alpha_{1}, \cdots, \alpha_{n} \in K$, então podemos escrever $\alpha_{i}=\beta_{i} \gamma^{-1}, 1 \leq i \leq n$, onde $\beta_{i}$ e $\gamma$ estão em $A$ e, $\gamma \mathcal{F} \subseteq A$. Reciprocamente, se $A$ é Noetheriano, todo ideal fracionário $\mathcal{F}$ é finitamente gerado, pois $\mathcal{F}$ é da forma $\alpha^{-1} \mathcal{I}$, onde $\mathcal{I}=\alpha \mathcal{F}$ é um ideal ordinário de $A$ que é finitamente gerado.

Um $A$-submódulo $\mathcal{F}$ de $K$ é dito ser um ideal invertível de $A$ se existe um submódulo $\mathcal{B}$ de $K$ tal que $\mathcal{F B}=A$. $\mathrm{O}$ módulo $\mathcal{B}$, quando existe, é único e igual a $(A: \mathcal{F}) ;$ pois

$$
\mathcal{B} \subseteq(A: \mathcal{F})=(A: \mathcal{F}) \mathcal{F B} \subseteq A \mathcal{B}=\mathcal{B}
$$

Todo ideal invertível $\mathcal{F}$ de $A$ é finitamente gerado e, portanto, um ideal fra- 
cionário. De fato, desde que $\mathcal{F}(A: \mathcal{F})=A$, existem $\alpha_{i} \in \mathcal{F}$ e $\beta_{i} \in(A: \mathcal{F})$, $1 \leq i \leq n$, tais que $\sum_{i=1}^{n} \alpha_{i} \beta_{i}=1$. Assim, para todo $\alpha \in \mathcal{F}$ temos $\alpha=\sum_{i=1}^{n}\left(\beta_{i} \alpha\right) \alpha_{i}$, onde cada $\beta_{i} \alpha \in A$, ou seja, $\mathcal{F}$ é gerado por $\alpha_{1}, \cdots, \alpha_{n}$.

Claramente todo ideal fracionário principal não nulo $(u)$ é invertível, e seu inverso é $\left(u^{-1}\right)$. É evidentemente, os ideais invertíveis de $A$ formam um grupo com respeito à multiplicação, cujo elemento identidade é $A=(1)$.

A próxima proposição nos mostra que ser invertível é uma propriedade local.

Proposição 2.1 Para um ideal fracionário $\mathcal{F}$ de um domínio $A$, são equivalentes:

i) $\mathcal{F}$ é invertivel;

ii) $\mathcal{F}$ é finitamente gerado e, para cada ideal primo $\mathcal{P}$ de $A, \mathcal{F}_{\mathcal{P}}$ é invertível;

iii) $\mathcal{F}$ é finitamente gerado e, para cada ideal maximal $\mathfrak{m}$ de $A, \mathcal{F}_{\mathrm{m}}$ é invertível.

Dem.: i) $\Rightarrow$ ii). Usando que a operação localização comuta com produtos e, com quociente quando $\mathcal{F}$ é finitamente gerado, obtemos $A_{\mathcal{P}}=(\mathcal{F}(A: \mathcal{F}))_{\mathcal{P}}=$ $=\mathcal{F}_{\mathcal{P}}\left(A_{\mathcal{P}}: \mathcal{F}_{\mathcal{P}}\right)$, para cada ideal primo $\mathcal{P}$ de $A$, o que implica $\left.i i\right)$.

ii) $\Rightarrow$ iii). Esta implicação é imediata.

iii) $\Rightarrow$ i). Seja $\mathcal{I}=\mathcal{F}(A: \mathcal{F})$, que é um ideal ordinário de $A$. Para cada ideal maximal $\mathfrak{m}$ de $A$, temos $\mathcal{I}_{\mathfrak{m}}=\mathcal{F}_{\mathfrak{m}}\left(A_{\mathfrak{m}}: \mathcal{F}_{\mathfrak{m}}\right)=A_{\mathfrak{m}}$, pois $\mathcal{F}$ é finitamente gerado e $\mathcal{F}_{\mathfrak{m}}$ é invertível. Assim $\mathcal{I}_{\mathfrak{m}}=A_{\mathfrak{m}}$, para cada ideal maximal $\mathfrak{m} \operatorname{de} A$ e, consequentemente $\mathcal{I}=A(\operatorname{ver}(3.8)$ em [Ati]), o que mostra $i)$. 
Para domínios locais temos:

Proposição 2.2 Seja $A$ um domínio local. Então $A$ é um anel de valorização discreta se, e somente se todo ideal fracionário não nulo de $A$ é invertível.

Dem.: Se $A$ é um domínio local que é um anel de valorização discreta então, como vimos na seção anterior, $A$ é um domínio Noetheriano local de dimensão $1 \mathrm{em}$ que cada ideal é uma potência do ideal maximal $\mathfrak{m}$, que é principal. Se $\mathfrak{m}=(\alpha)$ e $\mathcal{F} \neq 0$ é um ideal fracionário de $A$, então existe $\beta \in A$ tal que $\beta \mathcal{F} \subseteq A$. Assim $\beta \mathcal{F}$ é um ideal ordinário de $A$ que é uma potência de $\mathfrak{m}$, ou seja, $\beta \mathcal{F}=\left(\alpha^{r}\right)$, para algum $r \geq 0$. Consequentemente $\mathcal{F}=\left(\alpha^{r-s}\right)$, onde $s=v(\beta)$, o que mostra que $\mathcal{F}$ é invertível, pois é principal.

Reciprocamente, se todo ideal fracionário não nulo de $A$ é invertível então, em particular, todo ideal ordinário não nulo de $A$ é invertível e, portanto finitamente gerado. Assim $A$ é Noetheriano, ou seja, $A$ é um domínio Noetheriano local, com ideal maximal m. Afirmamos agora que cada ideal ordinário não nulo de $A$ é uma potência de $\mathfrak{m}$. Pois, se isso não ocorrer, $\sum$ o conjunto dos ideais ordinários não nulos de $A$ que não são potências de $\mathfrak{m}$, é não vazio. Ordenando $\sum$ por inclusão e usando o lema de Zorn, temos que $\sum$ tem um elemento maximal $\mathcal{I}$. Então $\mathcal{I} \subseteq \mathfrak{m}$ e $\mathcal{I} \neq \mathfrak{m}$. Assim, $\mathfrak{m}^{-1} \mathcal{I} \subseteq \mathfrak{m}^{-1} \mathfrak{m}=A$, ou seja, $\mathfrak{m}^{-1} \mathcal{I}$ é também um ideal ordinário (próprio) de $A \operatorname{com} \mathcal{I} \subseteq \mathfrak{m}^{-1} \mathfrak{m} \mathcal{I} \subseteq \mathfrak{m}^{-1} \mathcal{I}$. Mas $\mathcal{I} \neq \mathfrak{m}^{-1} \mathcal{I}$ pois caso contrário, $\mathcal{I}=\mathfrak{m} \mathcal{I}$ e, pelo lema de Nakayama teríamos $\mathcal{I}=(0)$. Logo, pela maximalidade de $\mathcal{I}$, temos que $\mathfrak{m}^{-1} \mathcal{I}$ é uma potência de $\mathfrak{m}$, o que contradiz o fato de $\mathcal{I} \in \sum$. Assim, cada ideal ordinário não nulo de $A$ é uma potência de m.

Se $\mathfrak{m}=\mathfrak{m}^{2}$, então $\mathfrak{m}=\mathfrak{m}^{n}$ para todo $n \geq 1 \mathrm{e}$, neste caso, $\mathfrak{m}$ é o único ideal primo não nulo de $A$, ou seja, $A$ tem dimensão 1 .

Se $\mathfrak{m} \neq \mathfrak{m}^{2}$, então seja $\alpha \in \mathfrak{m}$ tal que $\alpha \notin \mathfrak{m}^{2}$. Mas $(\alpha)=\mathfrak{m}^{r}$ para algum $r \geq 1$. Como $\alpha \notin \mathfrak{m}^{2}$, temos que $r<2$. Assim, $r=1$ e $\mathfrak{m}=(\alpha)$. Se $A$ tem 
dimensão maior que 1 então, existe um ideal primo não nulo $\mathcal{P}$ de $A, \mathcal{P} \neq \mathfrak{m}$, tal que $(0) \subseteq \mathcal{P} \subseteq \mathfrak{m}$. Como $\mathcal{P}$ é uma potência de $\mathfrak{m}=(\alpha)$, temos que $\mathcal{P}=\left(\alpha^{k}\right)$, para algum $k>0$. Como $\mathcal{P} \neq \mathfrak{m}$, temos que $\alpha \notin \mathcal{P}$ o que contradiz o fato de $\mathcal{P}$ ser primo, pois $\alpha^{k} \in \mathcal{P}$. Logo também neste caso, $A$ tem dimensão 1. Agora o resultado segue do teorema (1.5).

A versão "global" da proposição anterior é:

Teorema 2.3 Seja $A$ um domínio integral. Então $A$ é um domínio de Dedekind se, e somente se, todo ideal fracionário não nulo de $A$ é invertível.

Dem.: Seja $\mathcal{F} \neq 0$ um ideal fracionário de $A$. Desde que $A$ é Noetheriano temos que $\mathcal{F}$ é finitamente gerado. Para cada ideal primo não nulo $\mathcal{P}$ de $A$, temos que $\mathcal{F}_{\mathcal{P}}$ é um ideal fracionário não nulo do anel de valorização discreta $A_{\mathcal{p}}$. Assim, da proposição (2.2) segue que $\mathcal{F}_{\mathcal{P}}$ é invertível, para cada ideal primo não nulo $\mathcal{P}$ de $A$. Então de (2.1) temos que $\mathcal{F}$ é invertível.

Se todo ideal fracionário não nulo de $A$ é invertível então, como na demonstração da proposição anterior, temos que $A$ é Noetheriano. Mais ainda, $A$ tem dimensão 1, pois caso contrário, existe uma sequência de ideais primos $(0) \subseteq \mathcal{P} \subseteq \mathfrak{m}$ com $\mathcal{P} \neq(0), \mathcal{P} \neq \mathfrak{m}$ e $\mathfrak{m}$ maximal. Assim, pela correspondência entre os ideais de $A$ e de $A_{\mathrm{m}}$, temos que $A_{\mathrm{m}}$ é um domínio Noetheriano local de dimensão $>1$. Da proposição (2.1), temos também que cada ideal fracionário não nulo de $A_{\mathrm{m}}$ é invertível, o que contradiz o fato da dimensão de $A_{\mathfrak{m}}$ ser maior que 1. Logo $A$ é um domínio Noetheriano de dimensão 1 e, consequentemente, cada ideal primo não nulo é maximal.

Para cada ideal primo não nulo $\mathcal{P}$ de $A$, seja $\mathcal{J} \neq(0)$ um ideal ordinário de $A_{\mathcal{P}}$. Se $\mathcal{I}=\mathcal{J} \cap A$, então $\mathcal{I}$ é um ideal invertível de $A$ e, de (2.1) temos que 
$\mathcal{J}=\mathcal{I}_{\mathcal{P}}$ é um ideal invertível de $A_{\mathcal{P}}$. Observe que se cada ideal ordinário de um anel é invertível, então cada ideal fracionário deste anel também o é. Logo, para cada ideal primo $\mathcal{P} \neq 0$ de $A$, temos que $A_{\mathcal{P}}$ é um domínio Noetheriano de dimensão 1 em que cada ideal fracionário não nulo é invertível. Então, de (2.2) temos que $A_{\mathcal{P}}$ é um anel de valorização discreta, para cada ideal primo $\mathcal{P} \neq 0$ e, como queríamos, $A$ é um domínio de Dedekind.

Desde que os ideais invertíveis de um anel $A$ formam um grupo multiplicativo, temos como consequência imediata do teorema (2.3) que:

Corolário 2.4 Se A é um domínio de Dedekind, então os ideais fracionários não nulos de A formam um grupo com respeito à multiplicação.

O grupo dos ideais fracionários não nulos do domínio de Dedekind $A$ é chamado o grupo de ideais de $A$ e denotado por $\mathfrak{F}(A)$. Nesta terminologia, dos corolários (1.7) e (2.4) obtemos que $\mathfrak{F}(A)$ é um grupo (abeliano) livre, gerado pelos ideais primos não nulos de $A$. 


\section{Módulos e Lattices}

Nesta seção apresentaremos algumas propriedades de módulos sobre domínios de Dedekind, bem como introduziremos a noção de lattice e algumas propriedades básicas de lattices sobre tais domínios.

No que segue, em todo este trabalho, $A$ denotará um domínio de Dedekind com corpo de frações $K$ e $A^{*}$ o grupo das unidades de $A$. Mais ainda, a menos de menção contrária, os espaços vetoriais considerados serão $K$-espaços vetoriais bem como os módulos serão $A$-módulos.

Sejam $V$ um espaço vetorial de dimensão $n$ e $M$ um subconjunto de $V$ que é um $A$-módulo com as operações induzidas pela estrutura de espaço vetorial de $V$. Considere

$$
K M=\{\alpha x ; \alpha \in K, x \in M\} .
$$

Desde que $M$ é um $A$-módulo e $K$ é o corpo quociente de $A$, temos que

$$
K M=\left\{\alpha^{-1} x ; \alpha \in A, \alpha \neq 0 \text { e } x \in M\right\}
$$

Então $K M$ é um subespaço vetorial de $V$, na realidade $K M$ é o subespaço de $V$ gerado por $M$. Dados $\alpha \in K$ e $\mathcal{F} \in \mathfrak{F}(A)$ escrevemos

$$
\alpha M=\{\alpha x ; x \in M\}
$$


$\mathcal{F} M=\left\{\sum_{i \in \Delta} \beta_{i} x_{i} ; \beta_{i} \in \mathcal{F}, x_{i} \in M\right.$ onde $\Delta$ é um conjunto finito de índices $\}$, que são claramente $A$-módulos. Para estas operações com módulos temos as propriedades seguintes de fácil verificação.

Proposição 3.1 Sejam $M, N$ módulos, $\mathcal{F}, \mathcal{B}$ ideais fracionários de $A$ e $\alpha \in K$. Valem as seguintes propriedades:

i) $\alpha(M \cap N)=(\alpha M)(\alpha N)$

ii) $(\alpha A) M=\alpha M$

iii) $(\alpha \mathcal{F}) M=\alpha(\mathcal{F} M)$

iv) $(\mathcal{F}+\mathcal{B}) M=\mathcal{F} M+\mathcal{B} M$

v) $(\mathcal{F B}) M=\mathcal{F}(\mathcal{B} M)$

vi) $\mathcal{F}(M+N)=\mathcal{F} M+\mathcal{F} N$

vii) $K(M+N)=K M+K N$.

Dem.: Imediata.

Para um $A$-módulo $M$, o posto de $M \rho(M)$, é definido como sendo a dimensão do $K$-espaço vetorial $K M$ sobre $K$.

O próximo teorema nos dá uma decomposição dos $A$-módulos de torção finitamente gerados.

Teorema 3.2 Seja $M$ um módulo de torção finitamente gerado. Então $M$ tem uma decomposição da forma $M=\oplus_{\mathcal{P}} M(\mathcal{P})$, onde

$$
M(\mathcal{P})=\left\{x \in M ; \mathcal{P}^{k} x=0, \text { para } k \text { suficientemente grande. }\right\},
$$


para cada ideal primo não nulo $\mathcal{P}$ de $A$.

Dem.: Como $M$ é um $A$-módulo de torção finitamente gerado, o ideal de $A$ $\operatorname{Ann}(M)=\{\alpha \in A ; \alpha M=0\}$ é diferente de zero. De (1.7) temos que $\operatorname{Ann}(M)$ tem uma fatoração em potências de ideais primos não nulos de $A$, isto é, $\operatorname{Ann}(M)=\mathcal{P}_{1}^{m_{1}} \ldots \mathcal{P}_{s}^{m_{s}}$, onde $\mathcal{P}_{1}, \ldots, \mathcal{P}_{s}$ são ideais primos distintos não nulos de $A$ e $m_{1}>0, \ldots, m_{s}>0$. Para os ideais $\mathcal{I}_{j}=\mathcal{P}_{1}^{m_{1}} \ldots \mathcal{P}_{j-1}^{m_{j-1}} \mathcal{P}_{j+1}^{m_{j+1}} \ldots \mathcal{P}_{s}^{m_{s}}$ de $A, j=1, \ldots, s$, temos que $A=\sum_{j=1}^{s} \mathcal{I}_{j}$. De fato, se $\sum_{j=1}^{s} \mathcal{I}_{j} \neq A$, então $\mathcal{I}=\sum_{j=1}^{s} \mathcal{I}_{j}$ é um ideal próprio de $A$. Como $A$ é um domínio de Dedekind, de (2.3) temos que $\mathcal{I}$ é invertível e $A=\mathcal{I I}^{-1}=\mathcal{I}_{1} \mathcal{I}^{-1}+\cdots+\mathcal{I}_{s} \mathcal{I}^{-1}$. Para cada $j=1, \ldots, s$, 。 ideal fracionário $\mathcal{I}_{j} \mathcal{I}^{-1} \subseteq \mathcal{I I}^{-1}=A$, ou seja, é um ideal ordinário de $A$. Logo, se $\mathcal{P}$ é um ideal primo de $A$ que aparece na fatoração de $\mathcal{I}$, então da igualdade $A=\mathcal{I}_{1} \mathcal{I}^{-1}+\cdots+\mathcal{I}_{s} \mathcal{I}^{-1}$ segue que existe $j=1, \ldots, s$ tal que $\mathcal{P}$ não aparece na fatoração de $\mathcal{I}_{j} \mathcal{I}^{-1}$, ou seja, $\mathcal{P}$ aparece na fatoração de $\mathcal{I}_{j}$. Portanto $\mathcal{P}=\mathcal{P}_{\boldsymbol{i}}$ para algum $i \neq j, 1 \leq i \leq s$. Consequentemente, $\mathcal{I}_{k} \subseteq \mathcal{P}$ para todo $k \neq j$ e $\mathcal{I}_{j} \nsubseteq \mathcal{P}$, o que é um absurdo pois $\mathcal{I}=\mathcal{I}_{j}+\sum_{k \neq j} \mathcal{I}_{k} \subseteq \mathcal{P}$. Assim $A=\sum_{j=1}^{s} \mathcal{I}_{j}$.

Usando que $A=\sum_{j=1}^{s} \mathcal{I}_{j}$, temos que $M=\sum_{j=1}^{s}\left(\mathcal{I}_{j} M\right)$. Dado $j=1, \ldots, s$, temos que $\mathcal{P}_{j}^{m_{j}} \mathcal{I}_{j}=\operatorname{Ann}(M)$, ou seja, $M\left(\mathcal{P}_{j}\right) \supseteq \mathcal{I}_{j} M$. Consequentemente $M=\sum_{j=1}^{s} M\left(\mathcal{P}_{j}\right)$. Mostremos agora que esta soma é direta. Se $x \in M\left(\mathcal{P}_{1}\right) \cap$ $\cap\left(M\left(\mathcal{P}_{2}\right)+\cdots+M\left(\mathcal{P}_{s}\right)\right)$, então $\mathcal{P}_{1}^{m_{1}} x=0$ e $\mathcal{P}_{2}^{m_{2}} \ldots \mathcal{P}_{s}^{m_{s}} x=0$. Mas, de maneira similar ao feito acima, pode-se mostrar que $A=\mathcal{P}_{1}^{m_{1}}+\mathcal{P}_{2}^{m_{2}} \ldots \mathcal{P}_{s}^{m_{n}}$. Isso implica que $1 x=0$. Finalmente, para cada ideal primo não nulo $\mathcal{P}$ de $A$, que não aparece na decomposição do ideal $\operatorname{Ann}(M)$, temos que $M(\mathcal{P})=\{0\}$. Podemos então escrever $M=\oplus \mathcal{P} M(\mathcal{P})$, onde $\mathcal{P}$ percorre o conjunto dos ideais primos não nulos de $A$ como queríamos demonstrar. 
Sejam $T$ o $A$-módulo de torção $K / A$ e $M$ um $A$-módulo de torção. Definimos o $A$-módulo dual de $M$ por

$$
M^{*}=\operatorname{Hom}_{A}(M, T)
$$

Para tais módulos, quando $A$ é um domínio de Dedekind, temos:

Lema 3.3 Se $M$ é um $A$-módulo de torção finitamente gerado, então $M \cong M^{*}$ e existe um isomorfismo canônico de $A$-módulos $M \cong M^{* *}$.

Dem.: Desde que $(M \oplus N)^{*} \cong M^{*} \oplus N^{*}$, é suficiente mostrarmos a primeira afirmção para $M=A / \mathcal{I}$, onde $\mathcal{I} \neq 0$ é um ideal de $A$. Um homomorfismo $\lambda: A / \mathcal{I} \longrightarrow T$ é determinado pelo seu valor em $\overline{1} \in A / \mathcal{I}$. Como qualquer elemento de $\mathcal{I}^{-1} / A$ pode ser escolhido para ser $\lambda(\overline{1})$, temos o isomorfismo canônico

$$
(A / \mathcal{I})^{*} \longrightarrow \mathcal{I}^{-1} / A, \lambda \mapsto \lambda(\overline{1})
$$

Agora $\mathcal{I}^{-1} / A \cong A / \mathcal{I}$ como $A$-módulo pois, vale mais geralmente $\mathcal{I}_{1} / \mathcal{I}_{1} \mathcal{I}_{2} \cong A / \mathcal{I}_{2}$, $\mathcal{I}_{1}, \mathcal{I}_{2}$ ideais não nulos de $A$ (ver 22D de [OM]). Logo $M \cong M^{*}$.

Para demonstrarmos a segunda afirmação é suficiente mostrarmos que a aplicação canônica $i_{M}: M \longrightarrow M^{* *}$, onde $\left(i_{M} x\right) \lambda=\lambda(x)$, para todo $x \in M$, é injetora, pois segue da primeira afirmação que $M$ e $M^{* *}$ são isomorfos como $A$-módulos. Novamente podemos considerar que $M=A / \mathcal{I}$, onde $\mathcal{I} \neq 0$ é um ideal de $A$. Sejam $x \in M$ não nulo, ou seja, $x \in A-\mathcal{I}$ e $\mathcal{I}^{\prime}=\mathcal{I}+x A$. Escolhendo $\lambda(\overline{1}) \in\left(\mathcal{I}^{-1} / A\right)-\left(\mathcal{I}^{-1} / A\right)$, temos $\lambda(x) \in\left(x \mathcal{I}^{-1} / A\right)-\left(x \mathcal{I}^{-1} / A\right)$. Portanto $\lambda(x) \notin A$ e, então $\left(i_{M} x\right) \lambda \neq 0$. O que mostra que $i_{M}$ é injetora, como queríamos.

O próximo resultado sobre módulos sobre um domínios de Dedekind nos será útil no final da seção para caracterizarmos os módulos livre de torção. 
Lema 3.4 Se $M, N$ são $A$-módulos, $A$ um domínio de Dedekind e $M \otimes N$ é um A-módulo livre não nulo, então $M, N$ são projetivos.

Dem.: Dado um $A$-módulo $M$, existe um $A$-módulo livre $L$ e um homomorfismo sobrejetor de $A$-módulos $f: L \longrightarrow M$. Desde que $M \otimes N$ é livre, temos que o homomorfismo sobrejetor $f \otimes 1_{N}: L \otimes N \longrightarrow M \otimes N$ cinde, ou seja, existe um homomorfismo de módulos $g: M \otimes N \longrightarrow L \otimes N$ tal que $g \circ\left(f \otimes 1_{N}\right)=1_{L \otimes N}$. Então, $f \otimes 1_{N} \otimes 1_{M}: L \otimes N \otimes M \longrightarrow M \otimes N \otimes M$ também cinde, o que implica que $M \otimes N \otimes M$ é isomorfo a um somando direto do $A$-módulo livre $L \otimes N \otimes M$. Como $M \otimes N \otimes M \cong A^{s} \otimes M$, pois $M \otimes N$ é livre, temos que $M$ é isomorfo a um somando direto de $M \otimes N \otimes M$. Consequentemente, $M$ é isomorfo a um somando direto do $A$-módulo livre $L \otimes N \otimes M$, o que mostra que $M$ é projetivo. Mostrar que $N$ é projetivo é análogo.

Vamos agora introduzir a noção de lattice sobre $A$.

Definição 3.5 Sejam $V$ um espaço vetorial sobre $K$ e $\mathcal{L}$ um A-módulo com $\mathcal{L} \subseteq K$. Dizemos que $\mathcal{L}$ é uma lattice em $V$, com respeito à $A$, se existe uma base $\left\{x_{1}, \ldots, x_{n}\right\}$ de $V$ sobre $K$ tal que $\mathcal{L} \subseteq A x_{1}+\cdots+A x_{n}$. Dizemos que $\mathcal{L}$ é uma lattice sobre $V$ se $\mathcal{L}$ é uma lattice em $V$ tal que $K \mathcal{L}=V$.

Em particular se $\left\{x_{1}, \ldots, x_{n}\right\}$ é uma base de $V$, então $A x_{1}+\cdots+A x_{n}$ é uma lattice sobre $V$. O submódulo nulo $0=\{0\}$, será sempre considerado como uma lattice em $V$.

Proposição 3.6 Seja $\mathcal{L}$ uma lattice sobre um espaço vetorial $V$. Então um módulo $M \subseteq V$ é uma lattice em $V$ se, e somente se existe um elemento $\alpha$ não nulo em $A$ tal que $\alpha M \subseteq \mathcal{L}$. 
Dem.: Se $M$ é uma lattice em $V$, então existe uma base $\left\{x_{1}, \ldots, x_{n}\right\}$ de $V$ tal que

$$
M \subseteq A x_{1}+\cdots A x_{n}
$$

Desde que $\mathcal{L}$ é uma lattice sobre $V$, existem $y_{1}, \ldots, y_{n} \in \mathcal{L}$ linearmente independentes sobre $K$. Para $j=1, \ldots, n$, temos

$$
x_{j}=\sum_{i=1}^{n} a_{i j} y_{j}, \quad \text { com } a_{i j} \in K .
$$

Estes $a_{i j}$ geram um ideal fracionário de $A$. Assim, existe um $\alpha$ não nulo em $A$ tal que $\alpha a_{i j} \in A$ para todo $i, j=1, \ldots, n$. Então

$$
\alpha x_{j} \in A y_{1}+\cdots+A y_{n} \subseteq \mathcal{L}
$$

e isto implica que $\alpha M \subseteq \mathcal{L}$.

Reciprocamente, se existe $\alpha$ não nulo em $A$ tal que $\alpha M \subseteq \mathcal{L}$ então, desde que $\mathcal{L}$ é uma lattice em $V$, existe uma base $\left\{z_{1}, \ldots, z_{n}\right\}$ de $V$ tal que $\mathcal{L} \subseteq A z_{1}+\cdots+A z_{n}$. Logo

$$
M \subseteq \alpha^{-1} \mathcal{L} \subseteq A\left(\frac{z_{1}}{\alpha}\right)+\cdots+A\left(\frac{z_{n}}{\alpha}\right)
$$

ou seja, $M$ é uma lattice em $V$.

Proposição 3.7 Sejam $M$ um módulo e $U$ um subespaço vetorial de $V$, com $M \subseteq U \subseteq V$. Então $M$ é uma lattice em $V$ se, e somente se $M$ é uma lattice em $U$.

Dem.: Considere uma base $\left\{x_{1}, \ldots, x_{r}\right\}$ de $U$ e extenda-a para uma base $\left\{x_{1}, \ldots, x_{r}, x_{r+1}, \ldots, x_{n}\right\}$ de $V$. Sejam $\mathcal{L}^{\prime}=A x_{1}+\cdots+A x_{r}$ e $\mathcal{L}=A x_{1}+\cdots+A x_{n}$ lattices sobre $U$ e $V$ respectivamente, com $\mathcal{L}^{\prime} \subseteq \mathcal{L}$. Se $M$ é uma lattice em $U$, 
então existe $\alpha \neq 0$ em $A$ tal que $\alpha M \subseteq \mathcal{L}^{\prime} \subseteq \mathcal{L}$. Portanto, de (3.3), temos que $M$ é uma lattice em $V$.

Reciprocamente, se $M$ é uma lattice em $V$ tal que $M \subseteq U \subseteq V$, então $\alpha M \subseteq \mathcal{L}$ para algum $\alpha$ não nulo em $A$. Assim $\alpha M \subseteq \mathcal{L} \cap U=\mathcal{L}^{\prime}$ e, novamente pela proposição anterior, $M$ é uma lattice em $U$.

Quando não causar dúvidas sobre qual espaço vetoriais estamos considerando, mencionaremos simplesmente lattice para indicar lattice em $U, V$ etc. Segue imediatamente da definição de lattice que todo submódulo de uma lattice é também uma lattice. Em particular $\mathcal{L} \cap \mathcal{K}$ é uma lattice sempre que $\mathcal{L}$ e $\mathcal{K}$ são lattices em $V$. De (3.1) e (3.6) temos que $\alpha \mathcal{L}, \mathcal{F} \mathcal{L}$ e $\mathcal{L}+\mathcal{K}$ são lattices para qualquer $\alpha \in K, \mathcal{F}$ ideal fracionário de $A$ e $\mathcal{L}$ e $\mathcal{K}$ lattices. Claramente $A x$ e $\mathcal{F} x$ são lattices para qualquer $x$ em $V$ e $\mathcal{F} \in \mathfrak{F}(A)$. Assim, $\mathcal{F}_{1} z_{1}+\cdots+\mathcal{F}_{r} z_{r}$ é uma lattice, para quaisquer $\mathcal{F}_{i} \in \mathfrak{F}(A), z_{i} \in V, i=1, \ldots, r$. Em particular, todo $A$-módulo finitamente gerado em $V$ é uma lattice.

Sejam $V$ um espaço vetorial e $\mathcal{L}$ uma lattice. Para qualquer vetor $x$ não nulo em $K \mathcal{L}$ definimos o coeficiente de $x$ em $\mathcal{L}$ como sendo o conjunto

$$
\mathcal{F}_{x}=\{\alpha \in K ; \alpha x \in \mathcal{L}\}
$$

É imediato que $\mathcal{F}_{x}$ é um $A$-módulo em $K$ e, desde que $\mathcal{L}$ gera $K \mathcal{L}$, temos que $\mathcal{F}_{x} \neq\{0\}$. Agora, é fácil ver que

$$
\mathcal{F}_{x} x=\mathcal{L} \cap K x
$$

Assim, $\mathcal{F}_{x} x$ é uma lattice em $K x$. Logo $\alpha\left(\mathcal{F}_{x} x\right) \subseteq A x$, para algum $\alpha$ não nulo de $A$. Portanto $\alpha \mathcal{F}_{x} \subseteq A$, ou seja, $\mathcal{F}_{x}$ é de fato um ideal fracionário de $A$. Note que $\alpha \mathcal{F}_{\alpha x}=\mathcal{F}_{x}$, para todo $\alpha \in K^{*}$. É imediato que $\mathcal{F}_{x} \supseteq A$ se, e somente se $x \in \mathcal{L}$. 
Dizemos que $x$ é um vetor maximal de $\mathcal{L}$ se, e somente se

$$
\mathcal{L} \cap K x=A x .
$$

Quando todo ideal fracionário de $A$ é principal, temos que toda reta em $K \mathcal{L}$ contém um vetor maximal de $\mathcal{L}$. De fato, considere a reta $K y$ em $K \mathcal{L}$. Desde que $\mathcal{F}_{y}$ é um ideal fracionário de $A$, temos que $\mathcal{F}_{y}=\alpha A$, para algum $\alpha \in K$. Tomando $x=\alpha y$ temos $\mathcal{F}_{x}=A$. Assim $x$ é um vetor maximal de $\mathcal{L}$ que esta na reta $F y$.

Proposição 3.8 Sejam $\mathcal{L}$ uma lattice sobre $V, U$ um hiperplano em $V$ e $x_{0}$ um elemento de $V-U$. Então entre todos os elementos em $x_{0}+U$ existe pelo menos um cujo coeficiente com respeito à $\mathcal{L}$ é maximal. Seja $\mathcal{F}$ este coeficiente. Então para todo vetor $x_{0}+u_{0}, u_{0} \in U$ com coeficiente $\mathcal{F}$, temos

$$
\mathcal{L}=\mathcal{F}\left(x_{0}+u_{0}\right)+(\mathcal{L} \cap U)
$$

Dem.: Primeiramente afirmamos que

$$
\mathcal{F}=\left\{\alpha \in K ; \alpha x_{0} \in \mathcal{L}+U\right\}
$$

é um ideal fracionário em $K$. De fato, $\mathcal{F}$ é claramente um $A$-módulo não nulo em $K$ e, de (3.6) temos que existe um elemento $\beta \neq 0$ em $A$ tal que $\beta \mathcal{L} \subseteq A x_{0}+U$, pois $\mathcal{L}$ e $A x_{0}+U$ são lattices em $V$. Assim

$$
(\beta \mathcal{F}) x_{0} \subseteq \beta(\mathcal{L}+U) \subseteq \beta \mathcal{L}+U \subseteq A x_{0}+U
$$

Desde que $x_{0} \in V-U$, temos que $\beta \mathcal{F} \subseteq A$. O que mostra que $\mathcal{F}$ é um ideal fracionário de $A$ como requerido.

Agora, da definição de $\mathcal{F}$, temos que o coeficiente de qualquer vetor de $x_{0}+U$ com respeito a $\mathcal{L}$ está contido em $\mathcal{F}$. Assim a primeira parte da proposição será 
provada se pudermos encontrar um vetor $u$ em $U$ tal que $\mathcal{F}\left(x_{0}+u\right) \subseteq \mathcal{L}$, ou seja, $\mathcal{F} \subseteq \mathcal{F}_{x_{0}+u}$. Desde que $\mathcal{F F}^{-1}=A$, podemos encontrar uma expressão

$$
\alpha_{1} \beta_{1}+\cdots+\alpha_{r} \beta_{r}=1, \operatorname{com} \alpha_{i} \in \mathcal{F}, \beta_{i} \in \mathcal{F}^{-1}, i=1, \ldots, r .
$$

Agora, para cada $i=1, \ldots, r$, temos $\alpha_{i} x_{0} \in \mathcal{L}+U$, ou seja, existem $l_{1}, \ldots, l_{r}$ em $\mathcal{L}, u_{1}, \ldots, u_{r}$ em $U$ tais que $\alpha_{i} x_{0}=l_{i}+u_{i}$. Então, multiplicando $(*)$ por $x_{0}$, temos:

$$
x_{0}=\sum_{i=1}^{r} \beta_{i} l_{i}+\sum_{i=1}^{r} \beta_{i} u_{i} .
$$

Mas $\beta_{i} \mathcal{F} \subseteq A$ para cada $1 \leq i \leq r$. Assim,

$$
\mathcal{F}\left(x_{0}-\sum_{i=1}^{r} \beta_{i} u_{i}\right) \subseteq \mathcal{L} .
$$

Portanto encontramos $u \in U$ tal que $\mathcal{F}\left(x_{0}+u\right) \subseteq \mathcal{L}$ como queríamos.

Para $u_{0} \in U$, seja $\mathcal{F}$ o coeficiente de $x_{0}+u_{0}$ com respeito a $\mathcal{L}$. Temos então

$$
\mathcal{F}\left(x_{0}+u_{0}\right)+(\mathcal{L} \cap U) \subseteq \mathcal{L}
$$

Agora, desde que $U$ é um hiperplano em $V$ e $x_{0} \in V-U$, podemos escrever um elemento genérico de $\mathcal{L}$ na forma $\alpha\left(x_{0}+u\right)$, para algum $\alpha \in K$ e $u \in U$. Assim, $\alpha x_{0} \in \mathcal{L}+U$ e, da definição de $\mathcal{F}$, obtemos que $\alpha \in \mathcal{F}$. Logo,

$$
\alpha\left(u-u_{0}\right)=\alpha\left(x_{0}+u\right)-\alpha\left(x_{0}+u_{0}\right) \in \mathcal{L}
$$

pois $\mathcal{F}$ é o coeficiente de $x_{0}+u_{0}$ com respeito a $\mathcal{L}$. Obtemos então

$$
\alpha\left(x_{0}+u\right)=\alpha\left(x_{0}+u_{0}\right)+\alpha\left(u-u_{0}\right) \in \mathcal{F}\left(x_{0}+u_{0}\right)+(\mathcal{L} \cap U)
$$

como queríamos.

Vejamos agora um resultado de decomposição de lattices sobre domínios de Dedekind. 
Teorema 3.9 Sejam $A$ um domínio de Dedekind, $K$ seu corpo de frações, $V$ um espaço vetorial sobre $K, \mathcal{L}$ uma lattice sobre $V$ e $\left\{x_{1}, \ldots, x_{n}\right\}$ uma base de $V$ sobre $K$. Então existem uma base $\left\{y_{1}, \ldots, y_{n}\right\}$ de $V$ sobre $K$ com

$$
y_{i} \in K x_{1}+\cdots+K x_{i}, 1 \leq i \leq n
$$

e ideais fracionários $\mathcal{F}_{1}, \ldots, \mathcal{F}_{n}$ de $A$, tais que

$$
\mathcal{L}=\mathcal{F}_{1} y_{1}+\cdots+\mathcal{F}_{n} y_{n}
$$

Dem.: Seja $U$ o hiperplano, $K x_{1}+\cdots+K x_{n-1} \subseteq V$. Então, da proposição (3.8), temos

$$
\mathcal{L}=(\mathcal{L} \cap U)+\mathcal{F}_{n} y_{n}
$$

para algum ideal fracionário $\mathcal{F}_{n}$ e algum $y_{n} \in V-U$. Agora, o resultado segue por indução sobre $n=\operatorname{dim}_{K} V$.

Proposição 3.10 Sejam $\left\{x_{1}, \ldots, x_{n}\right\}$ uma base de $V$ sobre $K$ e $\mathcal{L}=\mathcal{F}_{1} x_{1}+$ $+\cdots+\mathcal{F}_{n} x_{n}, \operatorname{com} \mathcal{F}_{i} \in \mathfrak{F}(A)$. Então o coeficiente com respeito à $\mathcal{L}$ de qualquer vetor da forma $\alpha_{1} x_{1}+\cdots+\alpha_{r} x_{r}$, com $\alpha_{i} \in K^{*}$ é igual a $\left(\mathcal{F}_{1} \alpha_{1}^{-1}\right) \cap \cdots \cap\left(\mathcal{F}_{r} \alpha_{r}^{-1}\right)$. Em particular o coeficiente de $x_{i}$ é igual a $\mathcal{F}_{i}$.

Dem.: Seja $x=\alpha_{1} x_{1}+\cdots+\alpha_{r} x_{r}, \operatorname{com} \alpha_{i} \in K^{*}$. Temos então

$$
\mathcal{F}_{x}=\left\{\alpha \in K ; \alpha\left(\alpha_{1} x_{1}+\cdots+\alpha_{r} x_{r}\right) \in \mathcal{L}\right\}
$$

De $\alpha \alpha_{1} x_{1}+\cdots+\alpha \alpha_{r} x_{r} \in \mathcal{L}$, temos que $\alpha \alpha_{i} \in \mathcal{F}_{i}$, ou seja, $\alpha \in \mathcal{F}_{i} \alpha_{i}^{-1}$, para cada $i=1, \ldots, n$. Consequentemente, $\mathcal{F}_{x} \subseteq\left(\mathcal{F}_{1} \alpha_{1}^{-1}\right) \cap \cdots \cap\left(\mathcal{F}_{r} \alpha_{r}^{-1}\right)$.

Agora, se $\alpha \in\left(\mathcal{F}_{1} \alpha_{1}^{-1}\right) \cap \cdots \cap\left(\mathcal{F}_{r} \alpha_{r}^{-1}\right)$, então $\alpha \alpha_{i} \in \mathcal{F}_{i}$, para cada $i=1, \ldots, n$, o que implica que $\alpha\left(\alpha_{1} x_{1}+\cdots+\alpha_{r} x_{r}\right) \in \mathcal{L}$. Logo, $\mathcal{F}_{x} \supseteq\left(\mathcal{F}_{1} \alpha_{1}^{-1}\right) \cap \cdots \cap\left(\mathcal{F}_{r} \alpha_{r}^{-1}\right)$ o que mostra a proposição. 
Finalizamos esta seção, caracterizando os $A$-módulos livre de torção finitamente gerados sobre um domínio de Dedekind.

Teorema 3.11 Seja $M$ um A-módulo finitamente gerado, onde $A$ um domínio de Dedekind. Então $M$ é livre de torção se, e somente se $M$ é projetivo.

Dem.: Se $M$ é projetivo, então $M$ é livre de torção pois é somando direto de um módulo livre, que é livre de torção.

Reciprocamente, se $M$ é livre de torção então $K M$ é um espaço vetorial sobre $K$, pois $K M \cong S^{-1}(M), \operatorname{com} S=A-\{0\}$. Assim $M$ é uma lattice sobre $K M$ e de (3.9) existem uma base $\left\{e_{1}, \ldots, e_{n}\right\}$ de $V$ e ideais fracionários $\mathcal{F}_{1}, \ldots, \mathcal{F}_{n}$ de $A$ tais que $M=\mathcal{F}_{1} e_{1}+\cdots+\mathcal{F}_{n} e_{n}$. Agora, segue de (3.4) que cada $\mathcal{F}_{i}, i=1, \ldots, n$, é um $A$-módulo projetivo. Consequentemente $M$ é projetivo, pois é soma finita de módulos projetivos. 


\section{Sobre Decomposições de Lattices}

Nesta seção, continuaremos estudando decomposições de lattices, mais especificamente, daremos a noção de base em lattices, mudança de base e relações entre as decomposições de duas lattices em relação a uma mesma base do espaço vetorial que as contém.

Iniciaremos com uma consequência do teorema (3.9) que nos diz que uma lattice sobre $V$ é "quase livre".

Teorema 4.1 Seja $\mathcal{L}$ uma lattice sobre o espaço vetorial $V$. Então existem um ideal fracionário $\mathcal{F}$ de $A$ e uma base $\left\{z_{1}, \ldots, z_{n}\right\}$ de $V$ tais que

$$
\mathcal{L}=\mathcal{F} z_{1}+A z_{2}+\cdots+A z_{n}
$$

Dem.: De (3.9) temos que $\mathcal{L}=\mathcal{F}_{1} y_{1}+\cdots+\mathcal{F}_{n} y_{n}$, onde $\mathcal{F}_{i}$ são ideais fracionários de $A$ e $\left\{y_{1}, \ldots, y_{n}\right\}$ é uma base de $V$. Se $n=1$, temos $\mathcal{L}=\mathcal{F}_{1} y_{1}$. O caso $n \geq 3$ segue por aplicações sucessivas do caso $n=2$. Portanto, podemos assumir que $n=2$. Então, de (22:5) em [OM], podemos encontrar $\alpha_{1}, \alpha_{2} \in F$ tal que

$$
\alpha_{1} \mathcal{F}_{1}^{-1}+\alpha_{2} \mathcal{F}_{2}^{-2}=A
$$

Para $x=\alpha_{1} y_{1}+\alpha_{2} y_{2}$, de (3.10) e 22:4 em [OM], temos que o coeficiente de $x$ em $\mathcal{L}$ é igual a

$$
\left(\mathcal{F}_{1} \alpha_{1}^{-1}\right) \cap\left(\mathcal{F}_{2} \alpha_{2}^{-1}\right)=\left(\alpha_{1} \mathcal{F}_{1}^{-1}+\alpha_{2} \mathcal{F}_{2}^{-1}\right)^{-1}=A
$$


Assim por (3.8) segue que $\mathcal{L}=A x+\mathcal{B} y$, onde $B y=\mathcal{L} \cap A y$.

Dizemos que uma base $\left\{z_{1}, \ldots, z_{n}\right\}$ de $V$ é adaptada à lattice $\mathcal{L}$ se existem ideais fracionários $\mathcal{F}_{1}, \ldots, \mathcal{F}_{n}$ de $A$ tais que

$$
\mathcal{L}=\mathcal{F}_{1} z_{1}+\cdots+\mathcal{F}_{n} z_{n}
$$

Do teorema (3.9) temos que existe uma base para $K \mathcal{L}$ que é adaptada à $\mathcal{L}$, para qualquer lattice $\mathcal{L}$ em $V$, pois $\mathcal{L}$ é uma lattice sobre $K \mathcal{L}$.

Considere uma lattice $\mathcal{L}$ em $V$. Segue imediatamente do fato que $K$ é o corpo quociente de $A$ que um conjunto de vetores em $\mathcal{L}$ é linearmente independente sobre $A$ se, e somente se é linearmente independente sobre $K$. Assim um conjunto de vetores de $\mathcal{L}$ é linearmente independente maximal sobre $A$ se, e somente se ele é uma base para $K \mathcal{L}$. Em particular, quaisquer dois tais conjuntos devem conter o mesmo número de elementos. Este número é chamado o posto de $\mathcal{L}$ e denotado por $\rho(\mathcal{L})$. Portanto,

$$
\rho(\mathcal{L})=\operatorname{dim}_{K}(K \mathcal{L})
$$

Definição 4.2 Um conjunto de vetores de $V$ é dito ser uma base para $\mathcal{L}$ se é uma base no sentido de A-módulos, isto é, se é linearmente independente e gera $\mathcal{L}$ sobre $A$. Portanto $\left\{x_{1}, \ldots, x_{r}\right\}$ é uma base para $\mathcal{L}$ se, e somente se é uma base para $K \mathcal{L}$ tal que $\mathcal{L}=A x_{1}+\cdots+A x_{r}$.

Dizemos que uma lattice é livre quando admite uma base. Quaisquer duas bases de um lattice livre contém o mesmo número de elementos, este número é chamado a dimensão de $\mathcal{L}$ e é denotado por $\operatorname{dim} \mathcal{L}$. Assim,

$$
\operatorname{dim} \mathcal{L}=\operatorname{dim}_{K}(K \mathcal{L})=\rho(\mathcal{L})
$$


Do teorema (4.1) temos que, sobre um domínio de Dedekind $A$, cada lattice $\mathcal{L}$ é quase livre no sentido que

$$
\mathcal{L}=\mathcal{F}_{x_{1}}+A x_{2}+\cdots+A x_{r}
$$

com $\mathcal{F}$ um ideal fracionário de $A$ e $\left\{x_{1}, \ldots, x_{r}\right\}$ uma base de $K \mathcal{L}$. No caso em que $A$ é um domínio de Dedekind em que todo ideal fracionário é principal, ou mais especificamente num Domínio de ideais principais, temos do teorema (4.1) o seguinte corolário:

Corolário 4.3 Sejam $A$ um domínio de ideais principais e $V$ um $K$-espaço vetorial. Então toda lattice $\mathcal{L}$ sobre $V$ é da forma:

$$
\mathcal{L}=A z_{1}+\cdots+A z_{n}
$$

onde $\left\{z_{1}, \ldots, z_{n}\right\}$ é uma base de $V$.

Dem.: De (4.1) temos que existe uma base $\left\{z_{1}^{\prime}, z_{2}, \ldots, z_{n}\right\}$ de $V$ sobre $K$ e um ideal fracionário $\mathcal{F}$ de $A$ tal que $\mathcal{L}=\mathcal{F} z_{1}^{\prime}+A z_{2}+\cdots+A z_{n}$. Mas $\mathcal{F}$ é principal, ou seja, existe $\alpha \in K$ tal que $\mathcal{F}=A \alpha$. Logo $\left\{z_{1}=\alpha z_{1}^{\prime}, z_{2}, \ldots, z_{n}\right\}$ é uma base de $V$ tal que $\mathcal{L}=A z_{1}+A z_{2}+\cdots+A z_{n}$.

Considere duas lattices $\mathcal{L}$ e $\mathcal{L}^{\prime}$ sobre o mesmo espaço vetorial $V$ e sejam $\left\{x_{1}, \ldots, x_{n}\right\}$ e $\left\{y_{1}, \ldots, y_{n}\right\}$ bases de $V$ tais que

$$
\begin{aligned}
& \mathcal{L}=\mathcal{F}_{1} x_{1}+\cdots+\mathcal{F}_{n} x_{n}, \operatorname{com} \mathcal{F}_{i} \in \mathfrak{I}(A), \\
& \mathcal{L}^{\prime}=\mathcal{B}_{1} y_{1}+\cdots+\mathcal{B}_{n} y_{n}, \operatorname{com} \mathcal{B}_{i} \in \mathfrak{I}(A) .
\end{aligned}
$$

Sejam $y_{j}=\sum_{i} a_{i j} x_{i}$, e $x_{j}=\sum_{i} b_{i j} y_{i}$, as equações relacionando estas duas bases, ou seja, a matriz $\left(a_{i j}\right)$ é a inversa da matriz $\left(b_{i j}\right)$. 
Proposição 4.4 Nas condições acima, $\mathcal{L}^{\prime} \subseteq \mathcal{L}$ se, e somente se $a_{i j} \mathcal{B}_{j} \subseteq \mathcal{F}_{i}$, para todo $i, j=1, \ldots, n$.

Dem.: Temos $\mathcal{L}^{\prime} \subseteq \mathcal{L}$ se, e somente se $\mathcal{B}_{j} y_{j} \subseteq \mathcal{L}$ para todo $j=1, \ldots, n$, isto é, se, e somente se

$$
\mathcal{B}_{j}\left(a_{1 j} x_{1}+\cdots+a_{i j} x_{i}+\cdots+a_{n j} x_{n}\right) \subseteq \mathcal{F}_{1} x_{1}+\cdots+\mathcal{F}_{i} x_{i}+\cdots+\mathcal{F}_{n} x_{n},
$$

para $1 \leq j \leq n$. Desde que $\left\{x_{1}, \ldots, x_{n}\right\}$ é linearmente independente, temos que a inclusão acima é verdade se, e somente se $a_{i j} \mathcal{B}_{j} \subseteq \mathcal{F}_{i}$ para todo $i, j=1, \ldots, n$.

Como consequência da proposição (4.4), temos como caracterizar quando duas lattices em $V$ são iguais em termos dos ideais fracionários e de matrizes mudança de bases do espaço $V$.

Proposição 4.5 Se $\mathcal{L}^{\prime} \subseteq \mathcal{L}$ são lattices em $V$ como acima, então $\mathcal{L}^{\prime}=\mathcal{L}$ se, e somente se

$$
\mathcal{F}_{1} \ldots \mathcal{F}_{n}=\mathcal{B}_{1} \ldots \mathcal{B}_{n} \operatorname{det}\left(a_{i j}\right)
$$

Dem.: Se $\mathcal{L}^{\prime}=\mathcal{L}$, então de (4.4) temos que $a_{i j} \mathcal{B}_{j} \subseteq \mathcal{F}_{i}$, para todo $i, j=1, \ldots, n$, ou seja, $a_{i j} \in \mathcal{F}_{i} \mathcal{B}_{j}^{-1}$, para todo $i, j=1, \ldots, n$. Assim

$$
\operatorname{det}\left(a_{i j}\right)=\sum_{\sigma} \pm a_{1 \sigma(1)} \ldots a_{n \sigma(n)}
$$

onde $\sigma$ percorre o conjunto de todas as permutações de $\{1,2, \ldots, n\}$. Consequentemente

$$
\operatorname{det}\left(a_{i j}\right) \in \sum_{\sigma}\left(\mathcal{F}_{1} \mathcal{B}_{\sigma(1)}^{-1}\right) \ldots\left(\mathcal{F}_{n} \mathcal{B}_{\sigma(n)}^{-1}\right)=
$$




$$
\begin{gathered}
=\sum_{\sigma}\left(\mathcal{F}_{1} \ldots \mathcal{F}_{n}\right)\left(\mathcal{B}_{\sigma(1)}^{-1} \ldots \mathcal{B}_{\sigma(n)}^{-1}\right)= \\
=\sum_{\sigma}\left(\mathcal{F}_{1} \ldots \mathcal{F}_{n}\right)\left(\mathcal{B}_{\sigma(1)} \ldots \mathcal{B}_{\sigma(n)}\right)^{-1} \subseteq\left(\mathcal{F}_{1} \ldots \mathcal{F}_{n}\right)\left(\mathcal{B}_{1} \ldots \mathcal{B}_{n}\right)^{-1}
\end{gathered}
$$

Ou seja,

$$
\left(\mathcal{B}_{1} \ldots \mathcal{B}_{n}\right) \operatorname{det}\left(a_{i j}\right) \subseteq\left(\mathcal{F}_{1} \ldots \mathcal{F}_{n}\right)
$$

Usando agora que $\mathcal{L} \subseteq \mathcal{L}^{\prime}$ e repetindo o mesmo raciocínio, obtemos

$$
\left(\mathcal{F}_{1} \ldots \mathcal{F}_{n}\right) \operatorname{det}\left(b_{i j}\right) \subseteq\left(\mathcal{B}_{1} \ldots \mathcal{B}_{n}\right)
$$

Mas $\operatorname{det}\left(a_{i j}\right)$ é o inverso $\operatorname{de} \operatorname{det}\left(b_{i j}\right)$. Portanto $\left(\mathcal{F}_{1} \ldots \mathcal{F}_{n}\right)=\left(\mathcal{B}_{1} \ldots \mathcal{B}_{n}\right) \operatorname{det}\left(a_{i j}\right)$, como queríamos.

Reciprocamente, desde que $\mathcal{L}^{\prime} \subseteq \mathcal{L}$, é suficiente mostrar que $\mathcal{L} \subseteq \mathcal{L}^{\prime}$. Por (4.5) temos que $a_{i j} \in \mathcal{F}_{i} \mathcal{B}_{j}^{-1}$ para todo $i, j=1, \ldots, n$. Para cada $i, j$, a cofatora de $a_{i j}$ é igual a

$$
A_{i j}=\sum_{\substack{\sigma(i)=j\\}} a_{1 \sigma(1)} \ldots a_{i-1 \sigma(i-1)} a_{i+1 \sigma(i+1)} \ldots a_{n \sigma(n)} .
$$

Assim, $A_{i j} \mathcal{F}_{i} \mathcal{B}_{j}^{-1} \subseteq\left(\mathcal{F}_{1} \ldots \mathcal{F}_{n}\right)\left(\mathcal{B}_{1} \ldots \mathcal{B}_{n}\right)^{-1}=A \operatorname{det}\left(a_{i j}\right)$. Como $\left(b_{i j}\right)=\left(a_{i j}\right)^{-1}$, temos que $b_{i j}=\frac{A_{i j}}{\operatorname{det}\left(a_{i j}\right)}$ e, consequentemente, $b_{i j} \mathcal{F}_{i}=\frac{A_{i j}}{\operatorname{det}\left(a_{i j}\right)} \mathcal{F}_{i} \subseteq \mathcal{B}_{j}$, para cada $i, j=1, \ldots, n$. Logo, de (4.4) temos $\mathcal{L} \subseteq \mathcal{L}^{\prime}$, como queríamos.

Dizemos que uma matriz $\left(a_{i j}\right) \in M_{n}(K)$, é inteira com respeito a $A$ se, cada $a_{i j}$ está em $A$. Diremos também que $\left(a_{i j}\right)$ é unimodular se for inteira com $\operatorname{det}\left(a_{i j}\right)$ uma unidade de $A$. Se $\left(a_{i j}\right)$ é uma matriz unimodular, então $\left(b_{i j}\right) \in M_{n}(K)$, com $b_{i j}=\frac{A_{i j}}{\operatorname{det}\left(a_{i j}\right)}$, onde $A_{i j}$ é o cofator de $a_{i j}$, é a matriz inversa da matriz $\left(a_{i j}\right) \mathrm{e}$, como $A_{i j} \in A$ e $\operatorname{det}\left(a_{i j}\right) \in A^{*}$, temos que $b_{i j} \in A$, para todo $i, j=1, \ldots, n$, ou seja, $\left(b_{i j}\right)$ é também uma matriz unimodular. Da equação $b_{i j}=\frac{A_{i j}}{\operatorname{det}\left(a_{i j}\right)}$ temos 
que uma matriz $\left(a_{i j}\right) \in M_{n}(K)$ inteira, ou seja, $\left(a_{i j}\right) \in M_{n}(A)$ é unimodular se, e somente se $\left(a_{i j}\right)$ é invertível com inversa $\left(b_{i j}\right) \in M_{n}(A)$, isto é, inteira.

No caso de lattices temos que uma matriz mudança de base tem que ser unimodular, como mostra a próxima proposição.

Proposição 4.6 Seja $\mathcal{L} \subseteq V$ uma lattice livre com base $\left\{x_{1}, \ldots, x_{n}\right\}$. Considere os vetores $y_{1}, \ldots, y_{n} \in V$, onde

$$
y_{j}=\sum_{i} a_{i j} x_{i}, \operatorname{com} a_{i j} \in K
$$

Então $\left\{y_{1}, \ldots, y_{n}\right\}$ é uma base de $\mathcal{L}$ se, e somente se a matriz dos coeficientes $\left(a_{i j}\right)$ é unimodular.

Dem.: Decorre do fato que $\left(a_{i j}\right)$ será uma matriz mudança de base.

Corolário 4.7 Sejam $v_{1}, \ldots, v_{r}$ vetores em $V, \varepsilon \in A^{*}$ e $\alpha_{2}, \ldots, \alpha_{r}$ elementos em $A$. Se $\overline{v_{1}}=\varepsilon v_{1}+\alpha_{2} v_{2}+\cdots+\alpha_{r} v_{r}$, então $A v_{1}+A v_{2}+\cdots+A v_{r}=A \overline{v_{1}}+$ $+A v_{2}+\cdots+A v_{r}$.

Dem.: Neste caso a matriz mudança de base é

$$
\left(a_{i j}\right)=\left(\begin{array}{ccccc}
\varepsilon & 0 & 0 & \cdots & 0 \\
\alpha_{2} & 1 & 0 & \cdots & 0 \\
\alpha_{3} & 0 & 1 & \cdots & 0 \\
\vdots & \vdots & & \ddots & \vdots \\
\alpha_{r} & 0 & 0 & \cdots & 1
\end{array}\right),
$$

que é claramente unimodular. 
O próximo teorema que é o principal resultado desta seção, relaciona as decomposições de duas lattices em termos de uma mesma base do espaço vetorial dando origem ao que é chamado fatores invariantes de uma lattice em relação à outra.

Teorema 4.8 Sejam $\mathcal{L}$ e $\mathcal{L}^{\prime}$ lattices sobre um espaço vetorial $V \neq 0$. Então existe uma base $\left\{x_{1}, \ldots, x_{n}\right\}$ de $V$ tal que

$$
\begin{gathered}
\mathcal{L}=\mathcal{F}_{1} x_{1}+\cdots+\mathcal{F}_{n} x_{n}, \\
\mathcal{L}^{\prime}=\mathcal{F}_{1} \mathcal{R}_{1} x_{1}+\cdots+\mathcal{F}_{n} \mathcal{R}_{n} x_{n},
\end{gathered}
$$

onde $\mathcal{F}_{i}$ e $\mathcal{R}_{i}, i=1, \ldots, n$, são ideais fracionários de $A$ com

$$
\mathcal{R}_{1} \supseteq \mathcal{R}_{2} \supseteq \cdots \supseteq \mathcal{R}_{n}
$$

Os ideais fracionários $\mathcal{R}_{\boldsymbol{i}}$, determinados acima são únicos.

Dem.: Desde que $\mathcal{L}$ e $\mathcal{L}^{\prime}$ são lattices sobre $V$, de (3.6) temos que existe $\alpha \in A$, $\alpha \neq 0$, tal que $\alpha \mathcal{L}^{\prime} \subseteq \mathcal{L}$. Logo, trocando $\mathcal{L}^{\prime}$ por $\alpha \mathcal{L}^{\prime}$ se necessário, podemos supor que $\mathcal{L}^{\prime} \subseteq \mathcal{L}$. Como $\mathcal{L} K=V=\mathcal{L}^{\prime} K$, para cada $x \in V, x \neq 0$, podemos considerar $\mathcal{F}_{x}$ o coeficiente de $x$ em $\mathcal{L}$ e $\mathcal{B}_{x}$ o coeficiente de $x$ em $\mathcal{L}^{\prime}$, ambos ideais fracionários de $A$. Mais ainda, $\mathcal{B}_{x} \subseteq \mathcal{F}_{x}$ pois $\mathcal{L}^{\prime} \subseteq \mathcal{L}$. Com isso temos $\mathcal{R}_{x}=\mathcal{B}_{x} \mathcal{F}_{x}^{-1} \subseteq \mathcal{F}_{x} \mathcal{F}_{x}^{-1}=A$.

Agora, ordenando o conjunto dos $\mathcal{R}_{x}$, obtidos como acima, pela inclusão, podemos considerar $v \in V, v \neq 0$ tal que $\mathcal{R}_{v}$ seja maximal. Como $v \neq 0$, temos uma base de $V$ da forma $\left\{v, v_{2}, \ldots, v_{n}\right\}$. Seja $U$ o hiperplano gerado por $\left\{v_{2}, \ldots, v_{n}\right\}$. Como consequência do teorema (3.9), temos que existe um ideal fracionário $\mathcal{F}_{1}$ de $A$ tal que

$$
\mathcal{L}=\mathcal{F}_{1} v+\mathcal{L} \cap U
$$


Mais ainda, de (3.10) $\mathcal{F}_{1}$ é o coeficiente de $v$ em $\mathcal{L}$, ou seja, $\mathcal{F}_{1}=\mathcal{F}_{v}$. Logo $\mathcal{L}=\mathcal{F}_{v} v+(\mathcal{L} \cap U)$

Afirmamos que $\mathcal{B}_{v+u} \subseteq \mathcal{B}_{v}$, para todo $u \in U$. Caso contrário, aplicando a proposição (3.8) com $x_{0}=v$, temos que existe um elemento $v+u$ em $v+U$ tal que o coeficiente de $v+u$ em $\mathcal{L}^{\prime}$ é maximal, ou seja, $\mathcal{B}_{v+u}$ contém propriamente $\mathcal{B}_{v}$. Mas $\mathcal{F}_{v+u}(v+u) \subseteq \mathcal{L}=\mathcal{F}_{v} v+(\mathcal{L} \cap U)$, o que implica que $\mathcal{F}_{v+u} \subseteq \mathcal{F}_{v}$. Assim $\mathcal{R}_{v+u}=\frac{\mathcal{B}_{v+u}}{\mathcal{F}_{v+u}}$ que contém propriamente $\frac{\mathcal{B}_{v}}{\mathcal{F}_{v}}=\mathcal{R}_{v}$. Isto contradiz a maximalidade de $\mathcal{R}_{v}$, portanto temos que $\mathcal{B}_{v+u} \subseteq \mathcal{B}_{v}$, para todo $u$ em $U$.

Agora, novamente de (3.8), temos:

$$
\mathcal{L}^{\prime}=\mathcal{B}_{v} v+\left(\mathcal{L}^{\prime} \cap U\right)
$$

Usando indução, temos que o resultado vale para as lattices $\mathcal{L} \cap U$ e $\mathcal{L}^{\prime} \cap U$ sobre o espaço vetorial $U$. Obtemos então

$$
\begin{gathered}
\mathcal{L}=\mathcal{F}_{v} v+\left(\mathcal{F}_{2} v_{2}+\cdots+\mathcal{F}_{n} v_{n}\right), \\
\mathcal{L}^{\prime}=\mathcal{F}_{v} \mathcal{R}_{v} v+\left(\mathcal{F}_{2} \mathcal{R}_{2} v_{2}+\cdots+\mathcal{F}_{n} \mathcal{R}_{n} v_{n}\right),
\end{gathered}
$$

onde os $\mathcal{F}_{i}, \mathcal{R}_{i}, 2 \leq i \leq n$, são ideais fracionários de $A \operatorname{com} \mathcal{R}_{2} \supseteq \cdots \supseteq \mathcal{R}_{n}$. Mais ainda, $\mathcal{F}_{i}=\mathcal{F}_{v_{i}}$ e $\mathcal{R}_{i}=\mathcal{R}_{v_{i}}$ para todo $i=2, \ldots, n$. Assim, para a conclusão da primeira parte da demonstração do teorema, resta mostrarmos que $\mathcal{R}_{v} \supseteq \mathcal{R}_{2}=$ $=\mathcal{R}_{v_{2}}$. De (22:9) em [OM], temos que existem escalares não nulos $\alpha, \beta \in K$, tais que

$$
\left\{\begin{array}{l}
\alpha\left(\mathcal{F}_{v}^{-1} \mathcal{R}_{v}^{-1}\right)+\beta\left(\mathcal{F}_{2}^{-1} \mathcal{R}_{2}^{-1}\right)=A \\
\alpha \mathcal{F}_{v}^{-1}+\beta \mathcal{F}_{2}^{-1}=\mathcal{R}_{v}+\mathcal{R}_{2} .
\end{array}\right.
$$

Para $x=\alpha v+\beta v_{2}$, usando (3.10) e (22:4) de [OM] e a segunda identidade acima, obtemos

$$
\mathcal{F}_{x}=\left(\mathcal{F}_{v} \alpha^{-1}\right) \cap\left(\mathcal{F}_{2} \beta^{-1}\right)=\left(\alpha \mathcal{F}_{v}^{-1}+\beta \mathcal{F}_{2}^{-1}\right)^{-1}=\left(\mathcal{R}_{v}+\mathcal{R}_{2}\right)^{-1} .
$$


Similarmente, usando a primeira identidade, temos $\mathcal{B}_{x}=A$. Assim $\mathcal{R}_{x}=$ $=\mathcal{B}_{x} \mathcal{F}_{x}^{-1}=\mathcal{R}_{v}+\mathcal{R}_{2}$. Deste modo, pela escolha de $v$, temos que $\mathcal{R}_{x} \subseteq \mathcal{R}_{v}$ e, consequentemente, $\mathcal{R}_{2} \subseteq \mathcal{R}_{v}$, como queríamos.

Finalmente vamos mostrar a unicidade dos ideais fracionários $\mathcal{R}_{i}, i=1, \ldots, n$, ou seja, que estes ideais são independentes da base usada para obtê-los.

Suponhamos que $\mathcal{R}_{1}, \ldots, \mathcal{R}_{n}$ são os ideais fracionários obtidos usando a base $\left\{v_{1}, \ldots, v_{n}\right\}$ e $\mathcal{R}_{1}^{\prime}, \ldots, \mathcal{R}_{n}^{\prime}$ usando a base $\left\{v_{1}^{\prime}, \ldots, v_{n}^{\prime}\right\}$, ou seja,

$$
\left\{\begin{array}{l}
\mathcal{L}=\mathcal{F}_{1} v_{1}+\cdots+\mathcal{F}_{n} v_{n} \\
\mathcal{L}^{\prime}=\mathcal{F}_{1} \mathcal{R}_{1} v_{1}+\cdots+\mathcal{F}_{n} \mathcal{R}_{n} v_{n}
\end{array}\right.
$$

e

$$
\left\{\begin{array}{l}
\mathcal{L}=\mathcal{F}_{1}{ }_{1} v_{1}^{\prime}+\cdots+\mathcal{F}^{\prime}{ }_{n} v_{n}^{\prime} \\
\mathcal{L}^{\prime}=\mathcal{F}_{1}^{\prime}{ }_{1} \mathcal{R}_{1}^{\prime} v_{1}^{\prime}+\cdots+\mathcal{F}_{n}^{\prime} \mathcal{R}_{n}^{\prime} v_{n}^{\prime}
\end{array}\right.
$$

Suponhamos que $\mathcal{R}_{j}^{\prime} \neq \mathcal{R}_{j}$ para algum índice $j$. Seja $i$ o primeiro índice $(1 \leq i \leq n)$ tal que $\mathcal{R}_{i}^{\prime} \neq \mathcal{R}_{i}$. Então $\mathcal{R}_{\boldsymbol{i}} \subset \mathcal{R}_{\boldsymbol{i}}+\mathcal{R}^{\prime}{ }_{i}$ ou $\mathcal{R}_{i}^{\prime} \subset \mathcal{R}^{\prime}{ }_{i}+\mathcal{R}_{\boldsymbol{i}}$ (inclusões próprias). Sem perca de generalidade, podemos supor que $\mathcal{R}_{i}^{\prime} \subset \mathcal{R}_{i}^{\prime}+\mathcal{R}_{i}$.

Desde que $\mathcal{L}^{\prime} \subseteq \mathcal{L}$ e $\mathcal{L}^{\prime}$ e $\mathcal{L}$ são lattices sobre $V$, temos que a lattice $\mathcal{L}^{\prime \prime}=\mathcal{L}^{\prime}+\left(\mathcal{R}^{\prime}{ }_{i} \mathcal{L}\right)$ é também uma lattice sobre $V$ contida em $\mathcal{L}$.

Com relação a cada uma das bases separadamente temos

$$
\left\{\begin{array}{l}
\mathcal{L}=\mathcal{F}_{1} v_{1}+\cdots+\mathcal{F}_{i} v_{i}+\cdots+\mathcal{F}_{n} v_{n} \\
\mathcal{L}^{\prime \prime}=\mathcal{F}_{1}\left(\mathcal{R}_{1}+\mathcal{R}_{i}^{\prime}\right) v_{1}+\cdots+\mathcal{F}_{i}\left(\mathcal{R}_{i}+\mathcal{R}_{i}^{\prime}\right) v_{i}+\cdots+\mathcal{F}_{n}\left(\mathcal{R}_{n}+\mathcal{R}_{i}^{\prime}\right) v_{n}
\end{array}\right.
$$

e

$$
\left\{\begin{array}{l}
\mathcal{L}=\mathcal{F}^{\prime}{ }_{1} v_{1}^{\prime}+\cdots+\mathcal{F}^{\prime}{ }_{i} v_{i}^{\prime}+\cdots+\mathcal{F}_{n}^{\prime} v_{n}^{\prime} \\
\mathcal{L}^{\prime \prime}=\mathcal{F}^{\prime}{ }_{1}\left(\mathcal{R}^{\prime}{ }_{1}+\mathcal{R}^{\prime}{ }_{i}\right) v_{1}^{\prime}+\cdots+\mathcal{F}^{\prime}{ }_{i}\left(\mathcal{R}^{\prime}{ }_{i}+\mathcal{R}_{i}^{\prime}\right) v_{i}^{\prime}+\cdots+\mathcal{F}_{n}{ }_{n}\left(\mathcal{R}_{n}^{\prime}+\mathcal{R}^{\prime}\right) v_{n}^{\prime}
\end{array}\right.
$$


Então, para cada índice $\lambda$, com $1 \leq \lambda \leq i-1$, temos que o ideal fracionário obtido a partir de cada uma das bases é igual à $\mathcal{R}_{\lambda}$, pois como $\mathcal{R}_{\lambda}^{\prime} \supseteq \mathcal{R}_{i}^{\prime}(\lambda<i)$ e $\mathcal{R}_{\lambda}=\mathcal{R}_{\lambda}^{\prime}$, segue que $\mathcal{R}_{\lambda}+\mathcal{R}_{i}^{\prime}=\mathcal{R}_{\lambda}^{\prime}+\mathcal{R}_{i}^{\prime}=\mathcal{R}_{\lambda}^{\prime}=\mathcal{R}_{\lambda}$. Para $i \leq \lambda \leq n$, temos que os ideais fracionários obtidos são $\mathcal{R}_{\lambda}+\mathcal{R}_{i}^{\prime}$, à partir da base $\left\{v_{1}, \ldots, v_{n}\right\}$ e $\mathcal{R}_{\lambda}^{\prime}+\mathcal{R}_{i}^{\prime}=\mathcal{R}_{i}^{\prime}(i \leq \lambda)$, à partir da segunda base $\left\{v_{1}^{\prime}, \ldots, v_{n}^{\prime}\right\}$.

Agora, aplicando a proposição (4.5) para cada uma das decomposições de $\mathcal{L}$ obtemos $\mathcal{F}_{1} \ldots \mathcal{F}_{n}=\mathcal{F}^{\prime}{ }_{1} \ldots \mathcal{F}^{\prime}{ }_{n}$. Consequentemente, como $\mathcal{R}_{i} \subset \mathcal{R}_{\boldsymbol{i}}+\mathcal{R}^{\prime}{ }_{i}$, das duas decomposições da lattice $\mathcal{L}^{\prime \prime}$ obtemos que o produto dos ideais fracionários da primeira decomposição contém estritamente o produto dos ideais fracionários da segunda decomposição (ou em relação à segunda base), o que contradiz (4.5). Portanto para cada $i=1, \ldots, n, \mathcal{R}_{i}=\mathcal{R}^{\prime}{ }_{i}$, como queríamos.

Os únicos ideais fracionários $\mathcal{R}_{1} \supseteq \mathcal{R}_{2} \supseteq \cdots \supseteq \mathcal{R}_{n}$ do teorema acima são chamados os fatores invariantes de $\mathcal{L}^{\prime}$ em $\mathcal{L}$. Para cada $1 \leq i \leq n, \mathcal{R}_{i}$ é chamado o i-ésimo fator invariante de $\mathcal{L}^{\prime}$ em $\mathcal{L}$.

Observação 4.9 No caso em que $\mathcal{L}$ e $\mathcal{L}^{\prime}$ são duas lattices livres, segue de (4.6) e (4.8) que os fatores invariantes são ideais ordinários de $A$. 


\section{Relações entre Grupos de Witt}

$\mathrm{Da}$ teoria algébrica das forma quadráticas sobre anéis, temos relações entre o grupo de Witt dos espaços bilineares sobre um domínio de Dedekind $A$ e o grupo de Witt dos espaços bilineares sobre seu corpo de frações $K$. O nosso interesse, neste trabalho, é mais voltado para o ponto de vista aritmético da teoria de formas quadráticas. Nesta direção, apresentaremos a noção de formas bilineares simétricas em módulos de torção finitamente gerados sobre um domínio de Dedekind $A$ e, consequentemente, o grupo de Witt destas formas bilineares que, neste trabalho, chamaremos de grupo de Witt de torção de A. Apresentamos também relações, ou melhor, sequências exatas de grupos envolvendo os grupos de Witt citados acima.

$\mathrm{Na}$ teoria algébrica, estudamos formas bilineares simétricas $b: M \times M \rightarrow A$, onde $M$ é um módulo projetivo finitamente gerado sobre o anel $A$. No caso em que $A$ é um domínio de Dedekind, de (3.11), temos que os $A$-módulos projetivos finitamente gerados são precisamente os $A$-módulos livres de torção. Na teoria aritmética, é necessário estudar formas bilineares simétricas $b: M \times M \rightarrow T$, onde $T$ é o $A$-módulo de torção $K / A$ e $M$ é um $A$-módulo de torção finitamente gerado.

Definição 5.1 Seja $M$ um módulo finitamente gerado. Uma forma bilinear 
simétrica não singular (ou regular) $b$ em $M$ é uma função

$$
b: M \times M \longrightarrow T
$$

aditiva em ambas as variáveis com $b(x, y)=b(y, x)$, para todo $x, y \in M$, tal que $a$ função adjunta

$$
h: M \longrightarrow M^{*}=\operatorname{Hom}(M, T),
$$

definida por $h(x)=b(x$,$) é um isomorfismo. Neste caso, diremos que o par$ $(M, b)$ é um espaço bilinear.

Sejam $(M, b)$ um espaço bilinear e $N \subseteq M$ um submódulo. Definimos o complemento ortogonal de $N$ como sendo o submódulo de $M$,

$$
N^{\perp}=\{x \in M ; b(x, N)=0\} .
$$

As noções de soma ortogonal, subespaços, isometrias, elementos isotrópicos, anisotrópicos e subespaço totalmente isotrópico são definidas de maneira análoga ao caso algébrico e, podem ser vistas por exemplo em [Sch].

Um espaço bilinear $(M, b)$ é dito ser fracamente metabólico se existe um subespaço $N$ de $M$, tal que $N=N^{\perp}$.

Também de maneira análoga ao caso algébrico, obtemos o grupo de Witt de torção de $A, \mathcal{W T}(A)$, onde seus elementos são classes de isometrias de espaços bilineares $(M, b),[(M, b)]$, onde $M$ é um $A$-módulo de torção finitamente gerado $\mathrm{e}$, as classes dos espaços fracamente metabólicos são zero em $\mathcal{W} \mathcal{T}(A)$, ou seja, $\left[\left(M_{1}, b_{1}\right)\right]=\left[\left(M_{2}, b_{2}\right)\right]$ em $\mathcal{W} \mathcal{T}(A)$ se, e somente se existem espaços fracamente metabólicos $\left(N_{1}, b_{1}^{\prime}\right)$ e $\left(N_{2}, b_{2}^{\prime}\right)$ tais que

$$
\left(M_{1}, b_{1}\right) \perp\left(N_{1}, b_{1}^{\prime}\right) \simeq\left(M_{2}, b_{2}\right) \perp\left(n_{2}, b_{2}^{\prime}\right),
$$

onde o símbolo $\simeq$ significa "é isométrico à". O oposto de $[(M, b)]$ em $\mathcal{W} \mathcal{T}(A)$, é $[(M,-b)]$, desde que $(M, b) \perp(M,-b)$ é fracamente metabólico, pois $\Delta=\{x \oplus x ; x \in M\} \subseteq(M \oplus M, b \perp(-b))$ é tal que $\Delta^{\perp}=\Delta$. 
Lema 5.2 Seja $(M, b)$ um espaço bilinear. Para todo submódulo $N$ de $M$, temos $N=N^{\perp \perp}$.

Dem.: Por hipótese temos que existe $P \in M$ tal que $M=N \oplus P$. Logo, a sequência $M^{*} \rightarrow N^{*} \rightarrow 0$ é exata. Como $M$ é não singular, temos que $h: M \longrightarrow M^{*}$ é um isomorfismo. Através de composições obtemos a seguinte sequência exata $M \rightarrow N^{*} \rightarrow 0$. Note que $h: M \longrightarrow N^{*}$ leva $x \in M$ em $h(x) \in N^{*}$, onde $h(x)(y)=b(x, y)$ para todo $y \in N$, logo seu núcleo é $N^{\perp}$. Obtemos assim a sequência exata curta:

$$
0 \longrightarrow N^{\perp} \longrightarrow M \longrightarrow N^{*} \longrightarrow 0 .
$$

Como $N$ é projetivo temos que esta sequência exata cinde, ou seja, $M \cong N^{\perp} \oplus N^{*}$. O que mostra que $N^{\perp}$ é um subespaço de $M$.

Resta mostrarmos que $N=N^{\perp \perp}$. Para tanto consideremos o isomorfismo $h: M / N^{\perp} \rightarrow N^{*}$. Continuamos denotando por $h$ as aplicações que são definidas a partir da forma bilinear $b$. Este isomorfismo induz uma função bilinear não singular $b: N \times M / N^{\perp} \rightarrow T$; denotamos também por $b$ as restrições da forma bilinear $b$ a subespaços de $M$. Esta função bilinear aplicada a $N^{\perp}$ nos fornece $b: N^{\perp} \times M / N^{\perp \perp} \rightarrow T$. Por outro lado, temos o isomorfismo $M \cong\left(N^{\perp}\right)^{*} \oplus N$ que decorre dos isomorfismos:

$$
M^{*} \cong\left(N^{\perp}\right)^{*} \oplus N^{* *}, h: M \rightarrow M^{*} \text { e } N^{* *} \cong N .
$$

Assim, o isomorfismo $h: M / N \rightarrow\left(N^{\perp}\right)^{*}$ induz uma função bilinear não singular $b: N^{\perp} \times M / N \rightarrow T$. Agora, é fácil ver que $N \subseteq N^{\perp \perp}$. Então, vamos supor que $N \neq N^{\perp \perp} ; \operatorname{logo}$, existe $y \in N^{\perp \perp}-N$. Da primeira função bilinear obtida segue que $b(x, y)=0$ para todo $x \in N^{\perp}$, pois $y=0 \mathrm{em} M / N^{\perp \perp}$. Da segunda função bilinear segue que existe $x \in N^{\perp}$ tal que $b(x, y) \neq 0$, pois $y \neq 0$ em $M / N$ e $b$ é não singular. Chegamos assim a uma contradição. Portanto $N=N^{\perp \perp}$. 
O dois lemas seguintes terão importância no estabelecimento de relações entre $\mathcal{W}(K)$ e $\mathcal{W} \mathcal{T}(A)$

Lema 5.3 Seja $N$ um subespaço do espaço bilinear $(M, b) \operatorname{com} N \subset N^{\perp}$. Então b induz uma forma bilinear não singular $\bar{b}$ em $N^{\perp} / N$ de maneira que $(M, b) \oplus\left(N^{\perp} / N,-\bar{b}\right)$ é fracamente metabólica.

Dem.: Dado o espaço bilinear $(M, b)$ e $N \subseteq M$ com $N \subseteq N^{\perp}$, considere

$$
\bar{b}: \frac{N^{\perp}}{N} \times \frac{N^{\perp}}{N} \rightarrow T
$$

onde $\bar{b}(\bar{x}, \bar{y})=b(x, y)$, para todo $x, y \in N^{\perp}$. É fácil ver que $\vec{b}$ está bem definida. Mostremos que $\bar{b}$ é não singular. Seja $\bar{h}$ a adjunta de $\bar{b}$, ou seja, $\bar{h}: \frac{N^{\perp}}{N} \longrightarrow\left(\frac{N^{\perp}}{N}\right)^{*}$ e $\bar{h}(\bar{x})=\bar{b}(\bar{x}$,$) , para todo \bar{x} \in \frac{N^{\perp}}{N}$. Se $\bar{x}$ é um elemento do núcleo de $\bar{h}$, então, para todo $\bar{y} \in \frac{N^{\perp}}{N}$, temos $\bar{b}(\bar{x}, \bar{y})=0$, ou seja, $b(x, y)=0$, para todo $y \in N^{\perp}$. Assim $x \in N^{\perp \perp}=N$ pelo lema (5.2). Consequentemente $\bar{h}$ é injetora.

Mas, de (3.3), temos que os $A$-módulos $\frac{N^{1}}{N}$ e $\left(\frac{N^{\perp}}{N}\right)^{*}$ tem o mesmo comprimento. Então $\bar{h}$ é de fato um isomorfismo.

Agora, basta observar que o submódulo $M_{1}=\left\{(x, \bar{x}) ; x \in N^{\perp}\right\} \subseteq M \oplus\left(\frac{N^{\perp}}{N}\right)$ é tal que $M_{1}^{\perp}=M_{1}$, com respeito à forma $b \perp(-b)$.

Consideremos agora um espaço bilinerar simétrico (não singular) $(V, b)$ sobre $K$ e $\mathcal{L} \subseteq V$ uma lattice sobre $V$. É fácil ver que

$$
\mathcal{L}^{\sharp}=\{x \in V ; b(x, \mathcal{L}) \in A\}
$$

é também uma lattice em $V$, chamada a lattice dual de $\mathcal{L}$. Com esta terminologia temos: 
Lema 5.4 Sejam $(V, b)$ um espaço bilinear sobre $K$ e $\mathcal{L}$ uma lattice sobre $V$. Então:

i) Se $\left\{e_{1}, \ldots, e_{n}\right\}$ é uma base de $V$ tal que $\mathcal{L}=\mathcal{F}_{1} e_{1} \oplus \cdots \oplus \mathcal{F}_{n} e_{n}$, com $\mathcal{F}_{1}, \ldots, \mathcal{F}_{n}$ ideais fracionários de $A$, então $\mathcal{L}^{\sharp}=\mathcal{F}_{1}^{-1} e_{1}^{\prime} \oplus \cdots \oplus \mathcal{F}_{n}^{-1} e_{n}^{\prime}$, onde $\left\{e_{1}^{\prime}, \ldots, e_{n}^{\prime}\right\}$ é base dual de $\left\{e_{1}, \ldots, e_{n}\right\}$, ou seja, $b\left(e_{i}, e_{j}^{\prime}\right)=\delta_{i j}$, para todo $i, j=1, \ldots, n$.

ii) $S e \mathcal{L}_{1}$ e $\mathcal{L}_{2}$ são lattices sobre $V \operatorname{com} \mathcal{L}_{1} \subset \mathcal{L}_{2}$, então $\mathcal{L}_{2}^{\sharp} \subset \mathcal{L}_{1}^{\sharp}$.

iii) $\mathcal{L}^{\text {घH }}=\mathcal{L}$.

iv) Se $\mathcal{L}_{1}$ e $\mathcal{L}_{2}$ são duas lattices sobre $V$, então $\left(\mathcal{L}_{1} \cap \mathcal{L}_{2}\right)^{\sharp}=\mathcal{L}_{1}^{\sharp}+\mathcal{L}_{2}^{\sharp}$ e $\left(\mathcal{L}_{1}+\mathcal{L}_{2}\right)^{\sharp}=\mathcal{L}_{1}^{\sharp} \cap \mathcal{L}_{2}^{\sharp}$.

v) $\operatorname{Se} V=\mathcal{L} \otimes K$, com $\mathcal{L}$ um A-módulo, então $\mathcal{L}^{\sharp}=\mathcal{L}$.

Dem.: Observe que $x=\sum_{j=1}^{n} \alpha_{j} e_{j}^{\prime}$ está em $\mathcal{L}^{\sharp}$ se, e somente se para cada $i=1, \ldots, n$ e $\beta_{i} \in \mathcal{F}_{i}$, tivermos $b\left(x, \beta_{i} e_{i}\right)=\alpha_{i} \beta_{i}$ em $A$. Mas isto é verdade se, e somente se $\alpha_{i} \in \mathcal{F}_{i}^{-1}$, para todo $i=1, \ldots, n$, o que mostra $i$ ). A afirmação ii) segue diretamente da definição de $\mathcal{L}^{\sharp}$ e iii) segue diretamente de $i$ ).

Mostremos agora $i v$ ). Do Teorema dos divisores elementares (4.8), existe uma base $\left\{e_{1}, \ldots, e_{n}\right\}$ de $V$ e ideais fracionários $\mathcal{F}_{1}, \ldots, \mathcal{F}_{n}, \mathcal{B}_{1}, \ldots, ; \mathcal{B}_{n}$ de $A$ tais que $\mathcal{L}_{1}=\mathcal{F}_{1} e_{1} \oplus \cdots \oplus \mathcal{F}_{n} e_{n}$ e $\mathcal{L}_{2}=\mathcal{B}_{1} e_{1} \oplus \cdots \oplus \mathcal{B}_{n} e_{n}$. Assim, se $\left\{e_{1}^{\prime}, \ldots, e_{n}^{\prime}\right\}$ é base dual de $\left\{e_{1}, \ldots, e_{n}\right\}$, então de $\left.i\right)$ temos

$$
\begin{array}{r}
\left(\mathcal{L}_{1}+\mathcal{L}_{2}\right)^{\sharp}=\left(\left(\mathcal{F}_{1}+\mathcal{B}_{1}\right) e_{1} \oplus \cdots \oplus\left(\mathcal{F}_{n}+\mathcal{B}_{n}\right) e_{n}\right)^{\sharp}= \\
=\left(\mathcal{F}_{1}+\mathcal{B}_{1}\right)^{-1} e_{1}^{\prime} \oplus \cdots \oplus\left(\mathcal{F}_{n}+\mathcal{B}_{n}\right)^{-1} e_{n}^{\prime} \\
\text { e } \quad\left(\mathcal{L}_{1} \cap \mathcal{L}_{2}\right)^{\sharp}=\left(\mathcal{F}_{1} \cap \mathcal{B}_{1}\right)^{-1} e_{1}^{\prime} \oplus \cdots \oplus\left(\mathcal{F}_{n} \cap \mathcal{B}_{n}\right)^{-1} e_{n}^{\prime} .
\end{array}
$$

Agora o resultado segue usando que $\left(\mathcal{F}_{i}+\mathcal{B}_{i}\right)^{-1}=\mathcal{F}_{i}^{-1} \cap \mathcal{B}_{i}^{-1}$ e $\left(\mathcal{F}_{i} \cap \mathcal{B}_{i}\right)^{-1}=$ 
$=\mathcal{F}_{i}^{-1}+\mathcal{B}_{i}^{-1}$, para todo $i=1, \ldots, n$, concluindo assim a prova de $\left.i v\right),(\operatorname{ver}(\mathbf{2 2 : 4})$ em $[\mathrm{OM}])$.

Finalmente mostremos agora $v$ ). Se $\mathcal{L}$ é uma lattice sobre $\mathcal{L} \otimes K$, então usando o item i) existe uma base de $\mathcal{L} \otimes K,\left\{e_{1}, \ldots e_{n}\right\}$ e ideais fracionários $\mathcal{F}_{1}, \ldots, \mathcal{F}_{n}$ de $A$ tais que

$$
\mathcal{L}=\mathcal{F}_{1} e_{1}+\cdots+\mathcal{F}_{n} e_{n} \text { e } \mathcal{L}^{\sharp}=\mathcal{F}_{1}^{-1} e_{1}^{\prime}+\cdots+\mathcal{F}_{n}^{-1} e_{n}^{\prime},
$$

onde $\left\{e_{1}^{\prime}, \ldots, e_{n}^{\prime}\right\}$ é uma base dual de $\left\{e-1, \ldots, e_{n}\right\}$. Como cada $e_{i}=x_{i} \otimes \frac{1}{\alpha_{i}}$ $\operatorname{com} x_{i} \in \mathcal{L}$ e $\alpha_{i} \in A, i=1, \ldots, n$, temos que $e_{i}^{\prime}=x_{i}^{\prime} \otimes \alpha_{i} \operatorname{com} x_{i}^{\prime} \in \mathcal{L}$ tal que $b\left(x_{i}^{\prime}, x_{j}\right)=\delta_{i j}$, pois $b_{K}\left(x_{i} \otimes \frac{1}{\alpha_{i}}, x_{j}^{\prime} \otimes \alpha_{j}\right)=b\left(x_{i}, x_{j}^{\prime}\right) \otimes \frac{1}{\alpha_{i}} \alpha_{j}=1$. Assim, se $x \in \mathcal{L}^{\sharp}$ temos que $x=\sum_{i=1}^{n} a_{i} e_{i}^{\prime} \otimes \alpha_{i} \in \mathcal{L} \otimes K$, com $a_{i} \in A$ e, consequentemente temos que $\mathcal{L}^{\sharp} \subseteq \mathcal{L}$. A outra inclusão é, neste caso, trivial.

Corolário 5.5 Se $\mathcal{L}$ é uma lattice sobre $V$ livre, isto é, $\mathcal{L}=A e_{1}+\cdots+A e_{n}$, $\left\{e_{1}, \ldots, e_{n}\right\}$ uma base de $V$, então $\mathcal{L}^{\sharp}=A e_{1}^{\prime}+\cdots+A e_{n}^{\prime},\left\{e_{1}^{\prime}, \ldots, e_{n}^{\prime}\right\}$ base dual de $\left\{e_{1}, \ldots, e_{n}\right\}$.

Dem.: Segue imediatamente do item i) do lema anterior.

Observe que do item i) do lema anterior, obtemos que $\mathcal{L}^{\sharp}$ é também uma lattice sobre $V$. Assim, por (3.6), podemos sempre assumir que $\mathcal{L} \subseteq \mathcal{L}^{\sharp}$. De (5.4) temos que $\mathcal{L}=\mathcal{F}_{1} x_{1} \oplus \cdots \oplus \mathcal{F}_{n} x_{n}$ e $\mathcal{L}^{\sharp}=\mathcal{F}_{1}^{-1} x_{1}^{\prime} \oplus \cdots \oplus \mathcal{F}_{n}^{-1} x_{n}^{\prime}$, onde $\left\{x_{1}^{\prime}, \ldots, x_{n}^{\prime}\right\}$ é a base dual da base $\left\{x_{1}, \ldots, x_{n}\right\}$ de $V$ sobre $K$, ou seja, para cada $i=1, \ldots, n, x_{i}^{\prime}=\alpha_{i} x_{i}$, para algum $\alpha_{i} \in K$. Dado $x \in \mathcal{L}^{\sharp}$ temos que existem $\beta_{1}, \ldots, \beta_{n} \in K$ tais que $x=\sum_{i=1}^{n} \beta_{i} x_{i}^{\prime}=\sum_{i=1}^{n} \beta_{i} \alpha_{i} x_{i}$. Sejam $\mathcal{F}$ o ideal fracionário 
de $A$ gerado pelos $\beta_{i} \alpha_{i}, 1 \leq i \leq n$ e $\alpha \in A$ tal que $\alpha \mathcal{F} \subseteq A$. Para tal elemento $\alpha$ de $A$, temos $\alpha x \in \mathcal{L}$. Assim $\mathcal{L}^{\sharp} / \mathcal{L}$ é livre de torção.

Agora como na demonstração do lema (5.3), temos uma forma bilinear $b_{\mathcal{L}}$, induzida por $b$, no $A$-módulo de torção finitamente gerado $\mathcal{L}^{\sharp} / \mathcal{L}$.

$$
b_{\mathcal{L}}: \mathcal{L}^{\sharp} / \mathcal{L} \times \mathcal{L}^{\sharp} / \mathcal{L} \longrightarrow \mathcal{T},
$$

onde $b_{\mathcal{L}}(\bar{x}, \bar{y})=\overline{b(x, y)}$, para todo $x, y \in \mathcal{L}^{\sharp}$, ou seja, $b_{\mathcal{L}}(x+\mathcal{L}, y+\mathcal{L})=$ $=b(x, y)+A \in T=K / A$.

Com as considerações acima temos:

Proposição 5.6 A forma bilinear $b_{\mathcal{L}}$ é não singular, isto é, $\left(\mathcal{L}^{\sharp} / \mathcal{L}, b_{\mathcal{L}}\right)$ é um espaço bilinear e a função $(V, b) \longmapsto\left(\mathcal{L}^{\sharp} / \mathcal{L}, b_{\mathcal{L}}\right)$ induz um homomorfismo de grupos

$$
\partial: \mathcal{W}(K) \longrightarrow \mathcal{W T}(A)
$$

Dem.: Se $b_{\mathcal{L}}(\bar{x}, \bar{y})=0$, para todo $\bar{y} \in \mathcal{L}^{\sharp}$, então $b_{\mathcal{L}}(x, y) \in A$, para todo $y \in \mathcal{L}^{\sharp}$, o que implica que $x \in \mathcal{L}^{\sharp \sharp}=\mathcal{L}$ por (5.4). Logo $\bar{x}=0 \mathrm{em} \mathcal{L}^{\sharp} / \mathcal{L}$, ou seja, a função adjunta de $b_{\mathcal{L}}$ é injetora. Agora segue do lema (5.3), que $b_{\mathcal{L}}$ é não singular.

Para mostrarmos que $\partial$ está bem definida, consideremos o seguinte: se $\mathcal{L}_{0}, \mathcal{L}$ são lattices sobre $V \operatorname{com} \mathcal{L}_{0} \subseteq \mathcal{L}$, então de (5.4) temos que $\mathcal{L}^{\sharp} \subseteq \mathcal{L}_{0}^{\sharp}$. Como sempre podemos supor que $\mathcal{L} \subseteq \mathcal{L}^{\sharp}$, temos que o submódulo $\mathcal{L} / \mathcal{L}_{0}$ é totalmente isotrópico em $\mathcal{L}_{0}^{\sharp} / \mathcal{L}_{0}$. O complemento ortogonal de $\mathcal{L} / \mathcal{L}_{0}$ com respeito à $b_{\mathcal{L}_{0}}$ é $\left(\mathcal{L} / \mathcal{L}_{0}\right)^{\perp}=\left\{\bar{x} \in \mathcal{L}_{0}^{\sharp} / \mathcal{L}_{0} ; b(x, y) \in A\right.$, para todo $\left.y \in \mathcal{L}\right\}=\mathcal{L}^{\sharp} / \mathcal{L}_{0} \supseteq \mathcal{L} / \mathcal{L}_{0}$. Agora, aplicando (5.3) para $M=\mathcal{L}_{0}^{\sharp} / \mathcal{L}_{0}$ e $N=\mathcal{L} / \mathcal{L}_{0}$, temos que $N^{\perp} / N \cong \mathcal{L}^{\sharp} / \mathcal{L}$ e, consequentemente, os espaços $\left(\mathcal{L}^{\sharp} / \mathcal{L}, b_{\mathcal{L}}\right)$ e $\left(\mathcal{L}_{0}^{\sharp} / \mathcal{L}_{0}, b_{\mathcal{L}_{0}}\right)$ representam o mesmo elemento em $\mathcal{W} \mathcal{T}(A)$.

Agora, se $[(V, b)]=\left[\left(V^{\prime}, b^{\prime}\right)\right]$ em $\mathcal{W}(K)$ então, sem perda de generalidade podemos supor que $V=V^{\prime}$. Se $\mathcal{L}_{1}, \mathcal{L}_{2}$ são lattices em $V$ tais que $\partial[(V, b)]=$ 
$=\left[\left(\mathcal{L}_{1}^{\sharp} / \mathcal{L}_{1}, b_{\mathcal{L}_{1}}\right)\right]$ e $\partial\left[\left(V^{\prime}, b^{\prime}\right)\right]=\left[\left(\mathcal{L}_{2}^{\sharp} / \mathcal{L}_{2}, b_{\mathcal{L}_{2}}^{\prime}\right)\right]$ então, tomando $\mathcal{L}_{0}=\mathcal{L}_{1} \cap \mathcal{L}_{2}$ e aplicando o argumento usado acima, temos que $\partial$ está bem definida.

Finalmente, usando que $\frac{\mathcal{L}_{1}^{\sharp}}{\mathcal{L}_{1}} \oplus \frac{\mathcal{L}_{2}^{\sharp}}{\mathcal{L}_{2}} \cong \frac{\mathcal{L}_{1}^{\sharp}+\mathcal{L}_{2}^{\sharp}}{\mathcal{L}_{1} \cap \mathcal{L}_{2}}=\frac{\left(\mathcal{L}_{1} \cap \mathcal{L}_{2}\right)^{\sharp}}{\mathcal{L}_{1} \cap \mathcal{L}_{2}}$, é fácil ver que $\partial$ preserva soma ortogonal, ou seja, é um homomorfismo de grupos.

O nosso principal objetivo nesta seção é, usando o homomorfismo $\partial$ acima, estabelecermos uma sequência exata envolvendo os grupos de Witt $\mathcal{W}(A)$ dos espaços bilineares sobre $A, \mathcal{W}(K)$ dos espaços bilineares sobre $K$, e $\mathcal{W} \mathcal{T}(A)$ o grupo de Witt de torção de $A$. Para isso, necessitamos de mais um resultado auxiliar.

Lema 5.7 Seja $(L, b)$ um espaço bilinear sobre $A$. Se existe um submódulo $M$ de $L$ com $M^{\perp}=M$, então $M$ é um somando direto de $L$ e, em particular, $(L, b)$ é metabólico.

Dem.: Primeiro mostraremos que $L / M$ é um $A$-módulo livre de torção. De fato, se $x \in L-M$ é tal que $\alpha x \in M$, para algum $\alpha \neq 0$ de $A$ então, desde que $b$ é regular e $M^{\perp}=M$, existe $y \in M$ tal que $b(x, y) \neq 0$. Mas $0=b(\alpha x, y)=\alpha b(x, y)$, o que é uma contradição pois $A$ é um domínio. Portanto $L / M$ é um $A$-módulo livre de torção e, de (3.11) $L / M$ é um $A$-módulo projetivo. Consequentemente, a sequência exata de $A$-módulos $0 \longrightarrow M \longrightarrow L \longrightarrow L / M \longrightarrow 0$ cinde. Assim $L \cong M \oplus L / M$.

Vamos agora estabelecer uma relação entre os grupos de Witt.

Teorema 5.8 Seja A um domínio de Dedekind com corpo de frações $K$. Então a seguinte sequência de grupos de Witt é exata:

$$
0 \longrightarrow \mathcal{W}(A) \stackrel{i}{\rightarrow} \mathcal{W}(K) \stackrel{\partial}{\longrightarrow} \mathcal{W} \mathcal{T}(A)
$$


onde $i([(L, b)])=\left[\left(L \otimes K, b_{K}\right)\right]$ e $\partial([(V, b)])=\left[\left(\mathcal{L}^{\sharp} / \mathcal{L}, b_{\mathcal{L}}\right)\right]$.

Dem.: Dado $[(\mathcal{L}, b)] \in \mathcal{W}(A)$, podemos considerar $\mathcal{L}$ como uma lattice sobre o espaço vetorial $\mathcal{L} \otimes K$ e, neste caso, de (5.4) temos que $\mathcal{L}^{\sharp}=\mathcal{L}$. Mais ainda, como a definição de $\partial([(V, b)])$ não depende da escolha da lattice $\mathcal{L}$ sobre $V$, temos que $\partial \circ i([(\mathcal{L}, b)])=\partial\left(\left[\left(\mathcal{L} \otimes K, b_{K}\right)\right]\right)=\left[\left(\mathcal{L}^{\sharp} / \mathcal{L}, b_{\mathcal{L}}\right)\right]=0 \mathrm{em} \mathcal{W} \mathcal{T}(A)$, ou seja, $\partial \circ i=0$. Assim $\operatorname{Im}(i) \subseteq \operatorname{Ker}(\partial)$.

Reciprocamente, se $[(V, b)] \in \operatorname{Ker}(\partial)$, somando um espaço hiperbólico se necessário, podemos assumir que existe uma lattice $\mathcal{L}$ sobre $V$ tal que $\left(\mathcal{L}^{\sharp} / \mathcal{L}, b_{\mathcal{L}}\right)$ é fracamente metabólica. De (3.9) temos que $\mathcal{L}=\mathcal{F}_{1} e_{1} \oplus \cdots \oplus \mathcal{F}_{n} e_{n}$, onde $\left\{e_{1}, \ldots, e_{n}\right\}$ é uma base de $V$ e $\mathcal{F}_{1}, \ldots, \mathcal{F}_{n}$ são ideais fracionários de $A$. Trocando $\mathcal{L}$ por $\alpha \mathcal{L}$ se necessário, podemos assumir que $\mathcal{L} \subseteq N=A e_{1} \oplus \cdots \oplus A e_{n}$. Então $N$ é uma lattice sobre $V \operatorname{com} N K=V$. Logo de v) em (5.4) temos que $N=N^{\sharp}$. Agora, $[(V, b)]=i\left(\left[\left(N,\left.b\right|_{N}\right)\right]\right)$, o que mostra que que $\operatorname{Im}(i)=\operatorname{Ker}(\partial)$, ou seja, a sequência é exata em $\mathcal{W}(K)$.

Mostremos agora que $i$ é injetora. $\mathrm{Se}\left(\mathcal{L} \otimes K, b_{K}\right)$ é hiperbólico, então existe um subespaço $U$ de $\mathcal{L} \otimes K$ tal que $U=U^{\perp}$, ou seja, totalmente isotrópico maximal. Então $M=\mathcal{L} \cap U$ é um submódulo de $\mathcal{L}$ tal que $M=M^{\perp}$. Logo do lema (5.7) temos que $(\mathcal{L}, b)$ é metabólico. Mas no grupo de Witt $\mathcal{W}(A)$, todo metabólico é hiperbólico (ver (1.6.7) em [Sch]). Então $[(\mathcal{L}, b)]=0$ em $\mathcal{W}(A)$, o que mostra que $i$ é injetora como queríamos.

Vollamos novamenle nossa atenção para o grupo de Witt de torção $\mathcal{W T}(A)$. Se $[(M, b)] \in \mathcal{W} \mathcal{T}(A)$, então $M$ é um $A$-módulo de torção finitamente gerado e, de (3.2), temos que $M=\oplus_{\mathcal{P}} M(\mathcal{P})$, onde $\mathcal{P}$ percorre os ideais primos não nulos de $A$ e $M(\mathcal{P})=\left\{x \in M ; \mathcal{P}^{k} x=0\right.$, para $k$ suficientemente grande $\}$. Para 
cada ideal primo $\mathcal{P}$, o submódulo $M(\mathcal{P})$ é dito ser a componente $\mathcal{P}$-primária de $M$.

O próximo resultado mostra que esta decomposição de $M$ é ortogonal em relação a $b$.

Lema 5.9 Se $[(M, b)] \in \mathcal{W} \mathcal{T}(A)$, então a decomposição $M=\oplus_{\mathcal{P}} M(\mathcal{P})$ é uma decomposição ortogonal.

Dem.: Sejam $\mathcal{P} \neq \mathcal{Q}$ ideais primos não nulos de $A, x \in M(\mathcal{P})$ e $y \in M(\mathcal{Q})$. Então existem $r, s \geq 0$ tais que $\mathcal{P}^{r} x=\mathcal{Q}^{s} y=0$. Logo, para $k \geq \max \{r, s\}$ temos $\mathcal{P}^{k} x=\mathcal{Q}^{k} y=0$. Assim $b(x, y)$ é anulado por $\mathcal{P}^{k}$ e $\mathcal{Q}^{k}$ para $k$ suficientemente grande. Mas como $\mathcal{P}$ e $\mathcal{Q}$ são primos distintos, segue que $\mathcal{P}^{k}+\mathcal{Q}^{k}=A$. Consequentemente $b(x, y)=0$, o que mostra o lema.

Seja $b: M \times M \longrightarrow T$ uma forma bilinear simétrica não singular sobre o módulo de torção $M$. Para cada ideal primo não nulo $\mathcal{P}$ de $A$, seja $b_{\mathcal{P}}$ a restrição da forma bilinear $b$ à componente $\mathcal{P}$-primária $M(\mathcal{P})$ de $M$. Claramente a imagem de $b_{\mathcal{P}}$ está na componente $\mathcal{P}$-primária $T(\mathcal{P})$ do $A$-módulo de torção $T$, ou seja,

$$
b_{\mathcal{P}}: M(\mathcal{P}) \times M(\mathcal{P}) \longrightarrow T(\mathcal{P})
$$

Afirmamos que existe um isomorfismo de $A$-módulos

$$
T(\mathcal{P})=(K / A)(\mathcal{P}) \cong K / A_{\mathcal{P}}
$$

onde $A_{\mathcal{P}}$ é a localização de $A$ no ideal primo $\mathcal{P}$.

De fato, a projeção canônica $K / A \longrightarrow K / A_{\mathcal{P}}$ induz por restrição uma aplicação

$$
(K / A)(\mathcal{P}) \longrightarrow K / A_{\mathcal{P}}
$$


Observe que $x+A$ está em $(K / A)(\mathcal{P})$ se, e somente se existe $n \geq 0$ tal que $x \mathcal{P}^{n} \subseteq A$, ou seja, se $x \in \mathcal{P}^{-n}$. Agora $x+A$ está no núcleo da aplicação acima se, e somente se $x \in A_{\mathcal{P}}$, ou seja, $x \in A_{\mathcal{P}} \cap \mathcal{P}^{-n}=A$. Portanto a aplicação acima é injetora.

Seja $x \in K$ tal que $x+A_{\mathcal{P}} \neq A_{\mathcal{P}}$, ou seja, $x \notin A_{\mathcal{P}}$. Então $v_{\mathcal{P}}(x)=-n<0$, onde $v_{\mathcal{P}}$ é a valorização $\mathcal{P}$-ádica. Por (21:2) em [OM], existe $y \in \mathcal{P}^{-n}$ tal que $x-y \in A_{\mathcal{P}}$, ou seja, $x+A_{\mathcal{P}}=y+A_{\mathcal{P}}$ para algum $y \in \mathcal{P}^{-n}$. Assim $x+A_{\mathcal{P}}$ é a imagem de $y+A$ pela projeção canonica, $\operatorname{com} y+A$ em $(K / A)(\mathcal{P})$, o que mostra que $T(\mathcal{P}) \cong K / A_{\mathcal{P}}$. Finalmente, usando que $A / \mathcal{P}^{n} \cong A_{\mathcal{P}} / \mathcal{P}^{n} A_{\mathcal{P}}$, para cada ideal primo $\mathcal{P}$ de $A$ e (3.2) podemos identificar os $\mathcal{P}$-primários $A$-módulos de torção com os $A_{\mathcal{P}}$ módulos de torção. Juntamos todas estas considerações no seguinte teorema.

Teorema $5.10 \mathcal{W} \mathcal{T}(A) \cong \bigoplus_{\mathcal{P}} \mathcal{W} \mathcal{T}\left(A_{\mathcal{P}}\right)$, onde $\mathcal{P}$ percorre os ideais primos não nulos de A.

Dem.: Basta observar que cada $[(M, b)] \in \mathcal{W} \mathcal{T}(A)$ pode ser escrito na forma $\sum_{\mathcal{P}}\left[\left(M(\mathcal{P}), b_{\mathcal{P}}\right)\right] \in \sum_{\mathcal{P}} \mathcal{W} \mathcal{T}\left(A_{\mathcal{P}}\right)$.

Este resultado, e o teorema (5.8), nos leva a estudarmos o grupo de Witt de torção de um anel de valorização discreta que é o que faremos na próxima seção. 


\section{Grupos de Witt sobre Anéis de Valorização Discreta}

Em toda esta seção $A$ será um anel de valorização discreta, $K$ seu corpo de frações, $v$ a valorização de $K$ associada à $A, \mathcal{P}$ o único ideal maximal de $A$ e $\bar{K}=A / \mathcal{P}$ o corpo de classes residuais. Do teorema (1.5), temos que $\mathcal{P}$ é um ideal principal gerado por $\pi \in A$ tal que $v(\pi)=1$. Desde que todo anel de valorização discreta é um domínio de Dedekind, temos que os resultados da seção anterior valem para $A$. Nesta seção, apresentaremos alguns resultados mais refinados que possibilitarão conhecer mais, ou melhor, extendermos alguns resultados da seção anterior.

O próximo teorema relaciona o grupo de Witt de torção $\mathcal{W} \mathcal{T}(A)$ com o grupo de Witt do corpo de classes residuais $\mathcal{W}(\bar{K})$. Isto nos possibilita precisarmos o teorema (5.10).

Teorema 6.1 Existe um isomorfismo de grupos $\mathcal{W} \mathcal{T}(A) \cong \mathcal{W}(\bar{K})$ que depende da escolha do gerador do ideal maximal $\mathcal{P}$ de $A$.

Dem.: Seja $\pi$ um gerador de $\mathcal{P}$. Temos um homomorfismo de grupos

$$
\mathcal{W}(\bar{K}) \longrightarrow \mathcal{W} \mathcal{T}(A)
$$

induzido por:

$$
(b: V \times V \rightarrow \bar{K}=A / \mathcal{P}) \longrightarrow\left(\pi^{-1} b: V \times V \rightarrow T=K / A\right) .
$$


Mostraremos o teorema construindo um homomorfismo inverso. Agora vamos construir a inversa desta aplicação:

Seja $b: M \times M \longrightarrow K / A, M$ um módulo $\mathcal{P}$-primário. Se $\pi M=0$ então $M$ é um $\bar{K}$-espaço vetorial e $\pi b: M \times M \longrightarrow \bar{K}$ é um espaço bilinear sobre $\bar{K}$.

Se $\pi M \neq 0$ então, como $M$ é $\mathcal{P}$-torção, existe um $k \geq 2$ tal que $\pi^{k} M=0$ e $\pi^{k-1} M \neq 0$. O submódulo $M_{1}=\pi^{k-1} M$ de $M$ é totalmente isotrópico, pois $b\left(\pi^{k-1} x, \pi^{k-1} y\right)=\pi^{2(k-1)} b(x, y)=0$. Assim, de (5.3), temos $[(M, b)]=\left[\left(\overline{M_{1}}, \overline{b_{1}}\right)\right]$ em $\mathcal{W} \mathcal{T}(A), \operatorname{com} \overline{M_{1}}=M_{1}^{\perp} / M_{1}$ e $\pi^{k-1} \overline{M_{1}}=0$. Repetindo este processo um número finito de vezes, construímos $\overline{M_{r}}, r \geq 2, \operatorname{com}[(M, b)]=\left[\left(\overline{M_{r}}, \overline{b_{r}}\right)\right] \mathrm{e}$ $\pi \overline{M_{r}}=0$. Temos então que $\pi \overline{b_{r}}: \overline{M_{r}} \times \overline{M_{r}} \longrightarrow \bar{K}$ é um espaço bilinear sobre $\bar{K}$. Assim, usando (5.9), temos uma aplicação de $\mathcal{W} \mathcal{T}(A)$ em $\mathcal{W}(\bar{K})$, que respeita soma ortogonal, associando $[(M, b)] \in \mathcal{W} \mathcal{T}(A)$ à $\left[\left(\overline{M_{r}}\right), \overline{b_{r}}\right] \in \mathcal{W}(\bar{K})$.

Mais ainda, se $(M, b)$ é fracamente metabólico, então $\left(\overline{M_{1}}, \overline{b_{1}}\right), \ldots,\left(\overline{M_{r}}, \overline{b_{r}}\right)$ também o são pois, se $N$ é um șubmódulo de $M$ tal que $N=N^{\perp}$ com respeito à $b$, então o submódulo $\overline{N_{1}}=\left(N \cap M_{1}^{\perp}\right) /\left(N \cap M_{1}\right)$ de $\overline{M_{1}}$ é igual ao seu complemento ortogonal com respeito à $\overline{b_{1}}$ desde que $\left(N \cap M_{1}^{\perp}\right)^{\perp} \cap M_{1}^{\perp}=\left(N+M_{1}\right) \cap M_{1}^{\perp}=$ $=N \cap M_{1}^{\perp}$. Temos então um bem definido homomorfismo de grupos de $\mathcal{W}(A)$ em $\mathcal{W}(\bar{K})$ que é claramente o inverso pretendido.

A sequência exata de grupos de Witt apresentada no teorema (5.8), no caso em que $A$ é um anel de valorização discreta, pode ser completada para uma sequência exata curta, ou seja, vale o seguinte resultado:

Teorema 6.2 Se A é um anel de valorização discreta, então a sequência de grupos de Witt

$$
0 \longrightarrow \mathcal{W}(A) \stackrel{i}{\longrightarrow} \mathcal{W}(K) \stackrel{\partial}{\longrightarrow} \mathcal{W} \mathcal{T}(A) \longrightarrow 0
$$


é exata, onde $i([(M, b)])=\left[\left(M \oplus K, b_{K}\right)\right]$ e $\partial([(V, b)])=\left[\left(\mathcal{L}^{\sharp} / \mathcal{L}, b_{\mathcal{L}}\right)\right]$.

Dem.: Usando o fato que todo anel de valorização discreta é um domínio de Dedekind e o teorema (5.8), é suficiente mostrarmos que $\partial$ é sobrejetora. Do teorema (6.1) temos que todo elemento de $\mathcal{W} \mathcal{T}(A)$ pode ser representado por um espaço bilinear simétrico da forma $\left(\bar{K}^{n}, b\right)$, onde $b: \bar{K}^{n} \times \bar{K}^{n} \rightarrow \frac{\pi^{-1} A}{A} \subseteq \bar{K}$, com $\pi$ um gerador do ideal maximal $\mathcal{P}$ de $A$. Nesta representação $\bar{K}^{n}$ é considerado como um $A$-módulo de torção. Observe que $\pi b$ é uma forma bilinear simétrica sobre o corpo $\bar{K}$. Assim existe uma matriz simétrica $B \in \mathrm{M}_{n}(A)$ invertível, tal que $\bar{B} \in \mathrm{M}_{n}(\bar{K})$ representa a forma $\pi b$, ou seja, $\pi^{-1} \bar{B} \in \mathrm{M}_{n}(\bar{K})$ é a matriz de $b$. Agora, tomando $\left[\left(K^{n}, b^{\prime}\right)\right] \in \mathcal{W}(K)$ tal que $b^{\prime}$ é definida pela matriz $B$ e, escrevendo $K^{n}=K e_{1} \oplus \cdots \oplus K e_{n}$, temos que $\mathcal{L}=\mathcal{P} e_{1} \oplus \cdots \oplus \mathcal{P} e_{n}$ é uma lattice sobre $K^{n}$. Mais ainda, o $A$-módulo de torção $\mathcal{L}^{\sharp} / \mathcal{L}$ é tal que $\pi\left(\mathcal{L}^{\sharp} / \mathcal{L}\right)=0$. Assim, como na demonstração de (6.1) temos que $\mathcal{L}^{\sharp} / \mathcal{L}$ é um $\bar{K}$-espaço vetorial de dimensão $n$ e $\pi b_{\mathcal{L}}^{\prime}=\pi b$, pois é determinada pela matriz $\bar{B}$. Portanto, usando novamente o isomorfismo $\mathcal{W} \mathcal{T}(A) \cong \mathcal{W}(\bar{K})$, temos que $\partial\left(\left[\left(K^{n}, b^{\prime}\right)\right]\right)=\left[\left(\bar{K}^{n}, b\right)\right]$ como queríamos.

Um anel $A$ é dito ser 2-henseliano se satisfaz a propriedade: $a \in A^{*}$ é um quadrado se, e somente se $\bar{a} \in \bar{K}^{*}$ é um quadrado.

Da teoria de números algébricos, temos que todo anel de valorização discreta completo é 2-henseliano. Para tais anéis temos:

Teorema 6.3 Seja $A$ um anel de valorização discreta 2-henselliano tal que $\operatorname{car}(\bar{K}) \neq 2$. Então a sobrejeção canônica ${ }^{-}: A \longrightarrow \bar{K}$ indiz um isomorfismo de grupos $\mathcal{W}(A) \cong \mathcal{W}(\bar{K})$.

Dem.: Desde que $A$ é um anel local com $2 \in A^{*}$, temos que todo $A$-módulo 
projetivo finitamente gerado é livre e todo espaço bilinear simétrico $(M, b)$ sobre $A$ admite uma base ortogonal. A demonstração destes fatos que omitiremos aqui são de fácil compreenão e, podem ser encontrados em (1.6.3) e (1.6.4) em [Sch].

Se $\left\{e_{1}, \ldots, e_{n}\right\}$ é uma base ortogonal do espaço bilinear $(M, b)$, então denotaremos $b$ por $\left\langle\alpha_{1} ; \ldots, \alpha_{n}\right\rangle$ onde $\alpha_{i}=b\left(e_{i}, e_{i}\right)$, para $i=1, \ldots, n$. Nesta notação temos que $b$ é regular se, e somente se $\alpha_{i} \in A^{*}$ para todo $i=1, \ldots, n$.

Agora a sobrejeção canônica ${ }^{-}: A \longrightarrow \bar{K}$, induz um homomorfismo de grupos $\mathcal{W}(A) \longrightarrow \mathcal{W}(\bar{K})$, tal que $[(M, b)] \longmapsto[(\bar{M}, \bar{b})]$, onde se $b=\left\langle\alpha_{1}, \ldots, \alpha_{n}\right\rangle$, então $\bar{b}=\left\langle\bar{\alpha}_{1}, \ldots, \bar{\alpha}_{n}\right\rangle$. Para mostrarmos que este homomorfismo é bijetor, é suficiente mostrarmos que para $\alpha_{i}, \beta_{i} \in A^{*}, i=1, \ldots, n$, temos $\left\langle\alpha_{1}, \ldots, \alpha_{n}\right\rangle \simeq$ $\simeq\left\langle\beta_{1}, \ldots, \beta_{n}\right\rangle$ se $\left.\left\langle\bar{\alpha}_{1}, \ldots, \bar{\alpha}_{n}\right\rangle \simeq<\bar{\beta}_{1}, \ldots, \bar{\beta}_{n}\right\rangle$.

Se $\left.\left\langle\bar{\alpha}_{1}, \ldots, \bar{\alpha}_{n}\right\rangle \simeq<\bar{\beta}_{1}, \ldots, \bar{\beta}_{n}\right\rangle$, então existem $x_{i} \in A, i=1, \ldots, n$, tais que $\bar{\beta}_{1}=\bar{\alpha}_{1} \bar{x}_{1}{ }^{2}+\cdots+\bar{\alpha}_{n} \bar{x}_{n}{ }^{2}$. Desde que $\bar{\beta}_{1} \neq \overline{0}$, sem perda de generalidade podemos supor que $\bar{x}_{1} \neq \overline{0}$, ou seja, $x_{1} \in A^{*}$. Temos então que existe $a \in \mathcal{P}$, o ideal maximal de $A$, tal que $\alpha_{1} x_{1}^{2}+a=\beta_{1}-\left(\alpha_{2} x_{2}^{2}+\cdots+\alpha_{n} x_{n}^{2}\right)$, ou seja,

$$
x_{1}^{2}+\alpha_{1}^{-1} a=\alpha_{1}^{-1} \beta_{1}-\alpha_{1}^{-1}\left(\alpha_{2} x_{2}^{2}+\cdots+\alpha_{n} x_{n}^{2}\right) .
$$

$O$ elemento $\left(x_{1}^{2}+\alpha_{1}^{-1} a\right) \in A$ é tal que $\overline{x_{1}^{2}+\alpha_{1}^{-1} a}=\bar{x}_{1}{ }^{2} \neq \overline{0}$ e, como $A$ é 2henseliano, existe $x \in A^{*}$ tal que $x_{1}^{2}+\alpha_{1}^{-1} a=x^{2}$. Assim

$$
\beta_{1}=\alpha_{1} x^{2}+\cdots+\alpha_{n} x_{n}^{2}
$$

e, consequentemente, obtemos uma decomposição ortogonal $\left\langle\alpha_{1}, \ldots, \alpha_{n}\right\rangle \simeq$ $\simeq<\beta_{1}, \gamma_{2}, \ldots, \gamma_{n}>$, com $\gamma_{2}, \ldots, \gamma_{n} \in A^{*}$.

Como $\operatorname{car}(\bar{K}) \neq 2$, temos que o teorema do cancelamento de Witt vale para espaços bilineares sobre $\bar{K}$. Segue então que $\left\langle\overline{\gamma_{2}}, \ldots, \overline{\gamma_{n}}\right\rangle \simeq<\overline{\beta_{2}}, \ldots, \overline{\beta_{n}}>$. Agora a demonstração segue por indução. 
Mostraremos agora que se $A$ é um anel de valorização discreta 2-henseliano com: $\operatorname{car}(\bar{K}) \neq 2$, então a sequência exata do teorema (5.8) cinde, ou seja, $\mathcal{W}(K) \cong \mathcal{W}(\bar{K}) \oplus \mathcal{W}(\bar{K})$ obtendo assim o Teorema de Springer.

Como observado na demonstração de (6.2), toda forma bilinear simétrica não singular sobre $A$ é da forma $\left\langle\alpha_{1}, \ldots, \alpha_{n}\right\rangle, \operatorname{com} \alpha_{i} \in A^{*}=A-\mathcal{P}$. Como $K$ é um corpo de característica $\neq 2$, temos que toda forma bilinear simétrica sobre $K$ também admite uma base ortogonal (ver [Lam] ou (1.6.4) em [Sch]). Se $(V, b)$ é um espaço bilinear sobre $K$ e $b=\left\langle\alpha_{1}, \ldots, \alpha_{n}>\right.$, com $\alpha_{1}, \ldots, \alpha_{n} \in K-\{0\}$, multiplicando cada $\alpha_{i}$ por quadrados convenientes, podemos supor que $\alpha_{i} \in A$ para $i=1, \ldots, n$. Separando os $\alpha_{i} \in A-\mathcal{P}$ dos $\alpha_{i} \in \mathcal{P}$ e eliminando os quadrados, podemos escrever

$$
b=\left\langle\alpha_{1}, \ldots, \alpha_{k}, \pi \beta_{1}, \ldots, \pi \beta_{s}>, \operatorname{com} \alpha_{i}, \beta_{j} \in A-\mathcal{P}=A^{*} .\right.
$$

Uma consequência do fato que toda forma bilinear simétrica sobre $K$ pode ser expressa na forma acima, é que para conhecermos o homomorfismo $\partial: \mathcal{W}(K) \longrightarrow \mathcal{W} \mathcal{T}(A)$, é suficiente sabermos a ação de $\partial$ em espaços bilineares de dimensão $1 \mathrm{da}$ forma $\langle\alpha\rangle \mathrm{e}\langle\pi \alpha\rangle, \operatorname{com} \alpha \in A^{*}$.

Se $[(V, b)] \in \mathcal{W}(K)$ é tal que $b=\left\langle\alpha>\right.$, então $V=K x \operatorname{com} b(x, x)=\alpha \in A^{*}$ e, neste caso, $\mathcal{L}=A x$ é uma lattice sobre $V \operatorname{com} \mathcal{L}^{\sharp}=\mathcal{L}$, pois $\mathcal{L}^{\sharp}=A x^{\prime}$, com $x^{\prime}=\alpha^{-1} x \in A x$. Consequentemente $\partial([(V, b)])=0$.

Se $[(V, b)] \in \mathcal{W}(K)$ é tal que $b=<\pi \alpha>$, então $V=K y \operatorname{com} b(y, y)=$ $=\pi \alpha, \alpha \in A^{*}$. Também neste caso, $\mathcal{L}=A y$ é uma lattice sobre $V \operatorname{com} \mathcal{L}^{\sharp}=A y^{\prime}$, onde $y^{\prime}=\pi^{-1} \alpha^{-1} y$, ou seja, $\mathcal{L}^{\sharp}=\pi^{-1} \mathcal{L}$. Assim $\frac{\mathcal{L}^{\prime}}{\mathcal{L}}=\frac{\pi^{-1} \mathcal{L}}{\mathcal{L}}$ e $b_{\mathcal{L}}\left(\overline{\pi^{-1} y}, \overline{\pi^{-1} y}\right)=$ $=b\left(\pi^{-1} y, \pi^{-1} y\right)+A=\pi^{-1} \alpha+A$.

Agora, identificando $\mathcal{W T}(A) \operatorname{com} \mathcal{W}(\bar{K})$ pelo isomorfismo dado no teorema (6.1), podemos considerar que se $[(V, b)] \in \mathcal{W}(\bar{K})$ é tal que

$$
b=\left\langle\alpha_{1}, \ldots, \alpha_{k}, \pi \beta_{1}, \ldots, \pi \beta_{s}\right\rangle,
$$


com $\alpha_{i}, \beta_{j} \in A-\mathcal{P}, 1 \leq i \leq k$ e $1 \leq j \leq s$, então $\partial([(V, b)])=\partial([(b)])=$ $=\left[<\overline{\beta_{1}}, \ldots, \overline{\beta_{s}}>\right]=\left[\left(\bar{K},<\overline{\beta_{1}}, \ldots, \overline{\beta_{s}}>\right)\right] \in \mathcal{W}(\bar{K})$.

Considere agora, $\sigma: \mathcal{W}(\bar{K}) \longrightarrow \mathcal{W}(K)$ o homomorfismo de grupos tal que $\sigma([<\bar{\beta}>])=[\langle\pi \beta>]$, para todo $\bar{\beta} \neq \overline{0}$ em $\bar{K}$. É fácil ver que $\partial \circ \sigma$ é a identidade de $\mathcal{W}(\bar{K})$, o que mostra que se $A$ é um anel de valorização discreta 2-henseliano com $\operatorname{car}(\vec{K}) \neq 2$, a sequência exata de grupos

$$
0 \longrightarrow \mathcal{W}(A) \longrightarrow \mathcal{W}(K) \longrightarrow \mathcal{W} \mathcal{T}(A) \longrightarrow 0
$$

cinde. Assim, juntando os resultados dos teoremas (6.2) e (6.3), temos que neste caso, $\mathcal{W}(K) \cong \mathcal{W}(\bar{K}) \oplus \mathcal{W}(\bar{K})$.

Para finalizar, vamos calcular o anel de Witt dos espaços bilineares sobre $\mathbb{Z}$.

Do teorema (5.8) temos a seguinte sequência exata:

$$
0 \longrightarrow \mathcal{W}(\mathbb{Z}) \stackrel{i}{\longrightarrow} \mathcal{W}(\mathbb{Q}) \stackrel{\partial}{\longrightarrow} \mathcal{W} \mathcal{T}(\mathbb{Z}) .
$$

Logo, identificando $\mathcal{W}(\mathbb{Z})$ com $i(\mathcal{W}(\mathbb{Z}))$ temos que $\mathcal{W}(\mathbb{Z})$ é um subgrupo de $\mathcal{W}(\mathbb{Q})$ tal que $\frac{\mathcal{W}(\mathbb{Q})}{\mathcal{W}(\mathbb{Z})} \cong \operatorname{Im}(\partial) \subseteq \mathcal{W}(\mathbb{Z})$.

Dos teorema (5.10) e (6.1) temos que $\mathcal{W} \mathcal{T}(\mathbb{Z}) \cong \bigoplus_{\mathcal{P}} \mathcal{W}\left(\mathbb{F}_{\mathcal{P}}\right)$, onde $\mathcal{P}$ percorre o conjunto dos números primos. Sabemos também, da teoria algébrica das formas quadráticas sobre corpos, que $\mathcal{W}(\mathbb{Q}) \cong \mathcal{W}(\mathbb{R}) \oplus\left(\bigoplus_{\mathcal{P}} \mathcal{W}\left(\mathbb{F}_{\mathcal{P}}\right)\right)$, onde $\mathcal{P}$ percorre o conjunto dos números primos, (ver por exemplo (5.3.4) em [Sch]). Com estes isomorfismos, concluímos que $\mathcal{W}(\mathbb{Z})$ contém um subgrupo isomorfo à $\mathcal{W}(\mathbb{R}) \cong \mathbb{Z}$.

Vamos mostrar que de fato $\mathcal{W}(\mathbb{Z}) \cong \mathcal{W}(\mathbb{R})$. Para tanto, considerando a sequência exata de (6.2), temos que $\mathcal{W}(\mathbb{Z}) \cong \operatorname{Im}(i)=\operatorname{Ker}(\partial)$.

Dado $[(V, b)] \in \operatorname{Ker}(\partial)$, como na demonstração do teorema (5.8), temos que existe uma lattice $\mathcal{L}$ sobre $V$ tal que $\mathcal{L}^{\sharp}=\mathcal{L}$ e $[(V, b)]=i([(\mathcal{L}, b)])$. Agora, a forma bilinear $b$ sobre $\mathbb{Q}$ é da forma $b=\left\langle\alpha_{1}, \ldots, \alpha_{n}\right\rangle$, com $\alpha_{i} \in \mathbb{Z}, i=1, \ldots, n$. 
Seja $\left\{e_{1}, \ldots, e_{n}\right\}$ uma base de $\mathcal{L}$ sobre $\mathbb{Z}$ correspondente a esta decomposição de $b$, ou seja, $b\left(e_{i}, e_{j}\right)=0$, se $i \neq j$ e $b\left(e_{i}, e_{i}\right)=\alpha_{i}$, para todo $i, j=1, \ldots, n$.

$\operatorname{Se}\left\{e_{1}^{\prime}, \ldots, e_{n}^{\prime}\right\}$ é a base dual de $\left\{e_{1}, \ldots, e_{n}\right\}$, então cada $e_{i}^{\prime}=\beta_{i} e_{i}, \operatorname{com} \beta_{i} \in \mathbb{Q}$. De (5.4) temos que $\mathcal{L}^{\sharp}=\mathbb{Z} e_{1}^{\prime} \oplus \cdots \oplus \mathbb{Z} e_{n}^{\prime}$. Assim, para cada $i=1, \ldots, n$, $e_{\boldsymbol{i}}^{\prime}=\beta_{\boldsymbol{i}} e_{\boldsymbol{i}} \in \mathcal{L}^{\sharp}=\mathcal{L}$, o que implica que $\beta_{\boldsymbol{i}} \in \mathbb{Z}$ para todo $i=1, \ldots, n$.

Agora, para cada $i=1, \ldots, n, 1=b\left(e_{i}^{\prime}, e_{i}\right)=b\left(\beta_{i} e_{i}, e_{i}\right)=\beta_{i} \alpha_{i}$. Consequentemente $\alpha_{i}= \pm 1,1 \leq i \leq n$.

Mostramos então que $\operatorname{Ker}(\partial)$ é o subgrupo de $\mathcal{W}(\mathbb{Q})$ gerado aditivamente pelos elementos $[<1>]$ e $[<-1>]$ que é isomorfo à $\mathcal{W}(\mathbb{R})$, como queríamos. 


\section{Bibliografia}

[Ati] ATIYAH, M. F. and MACDOANLD, I. G., Introduction to Commutative Algebra, Addison-Wesley Publ. Comp., 1969.

[Bal] BALCERZYK, S. and JÓZEFIAK, T., Commutative Noetherian and Krull Rings, Ellis Horwood Series in Math. and its applications, 1989.

[OM] O'MEARA, O. T., Introduction to Quadratic Forms, A Series of Comp. Studies in Math. 117, Springer-Verlag, 1963.

[Lam] LAM, T. Y., The Algebraic Theory of Quadratic Forms, Math. Lecture Note Series, 1973.

[Sch] SCHARLAU, W., Quadratic and Hermitian Forms, A Series of Comp. Studies in Math. 270, Springer-Verlag, 1985.

[Mil] MILNOR, J. and HUSEMOLLER, D., Symmetric Bilinear Forms, E. M. G. 73, Springer-Verlag, 1973. 